\title{
LEAP2 changes with body mass and food intake in humans and mice
}

\author{
Bharath K. Mani, ${ }^{1,2,3}$ Nancy Puzziferri, ${ }^{4,5}$ Zhenyan He, ${ }^{1}$ Juan A. Rodriguez, ${ }^{1,2,3}$ Sherri Osborne-Lawrence, ${ }^{1,2,3}$ Nathan P. Metzger, ${ }^{1,2,3}$ \\ Navpreet Chhina, ${ }^{6,7}$ Bruce Gaylinn, ${ }^{8}$ Michael O. Thorner, ${ }^{8}$ E. Louise Thomas, ${ }^{9}$ Jimmy D. Bell, ${ }^{9}$ Kevin W. Williams, ${ }^{1}$ \\ Anthony P. Goldstone, ${ }^{6,7}$ and Jeffrey M. Zigman ${ }^{1,2,3}$ \\ 'Division of Hypothalamic Research, ${ }^{2}$ Division of Endocrinology \& Metabolism, Department of Internal Medicine, ${ }^{3}$ Department of Psychiatry, and ${ }^{4}$ Department of Surgery, UT Southwestern Medical \\ Center, Dallas, Texas, USA. ' Department of Surgery, Veterans Administration North Texas Heath Care System, Dallas, Texas, USA. ${ }^{6}$ PsychoNeuroEndocrinology Research Group, Neuropsychopharmacology \\ Unit, Centre for Psychiatry, and ' Computational, Cognitive and Clinical Neuroimaging Laboratory, Division of Brain Sciences, Imperial College London, Hammersmith Hospital, London, United Kingdom. \\ ${ }^{8}$ Department of Endocrinology, University of Virginia, Charlottesville, Virginia, USA. ${ }^{9}$ Research Centre for Optimal Health, University of Westminster, London, United Kingdom.
}

\begin{abstract}
Acyl-ghrelin administration increases food intake, body weight, and blood glucose. In contrast, mice lacking ghrelin or ghrelin receptors (GHSRs) exhibit life-threatening hypoglycemia during starvation-like conditions, but do not consistently exhibit overt metabolic phenotypes when given ad libitum food access. These results, and findings of ghrelin resistance in obese states, imply nutritional state dependence of ghrelin's metabolic actions. Here, we hypothesized that liver-enriched antimicrobial peptide-2 (LEAP2), a recently characterized endogenous CHSR antagonist, blunts ghrelin action during obese states and postprandially. To test this hypothesis, we determined changes in plasma LEAP2 and acyl-ghrelin due to fasting, eating, obesity, Roux-en-Y gastric bypass (RYCB), vertical sleeve gastrectomy (VSC), oral glucose administration, and type 1 diabetes mellitus (T1DM) using humans and/or mice. Our results suggest that plasma LEAP2 is regulated by metabolic status: its levels increased with body mass and blood glucose and decreased with fasting, RYCB, and in postprandial states following VSC. These changes were mostly opposite of those of acyl-ghrelin. Furthermore, using electrophysiology, we showed that LEAP2 both hyperpolarizes and prevents acyl-ghrelin from activating arcuate NPY neurons. We predict that the plasma LEAP2/acyl-ghrelin molar ratio may be a key determinant modulating acyl-ghrelin activity in response to body mass, feeding status, and blood glucose.
\end{abstract}

\section{Introduction}

Ghrelin is a mainly stomach-derived hormone that helps the body respond to changes in metabolic state by engaging growth hormone secretagogue receptors (GHSRs, also known as ghrelin receptors) expressed by neuronal circuits that regulate food intake, body weight, and blood glucose. (1-4). While ghrelin is found in circulation as both acyl-ghrelin and desacyl-ghrelin, only acyl-ghrelin, which receives its unique posttranslational acylation via interaction with ghrelin- $O$-acyltransferase (GOAT), binds GHSRs with high affinity $(5,6)$. Desacyl-ghrelin nonetheless has some biological activity, in some instances opposing acyl-ghrelin actions, although its mechanism of action at physiological levels appears GHSR independent (7-9).

Plasma acyl-ghrelin is regulated at least in part by metabolic status. In both humans and rodents, plasma acyl-ghrelin increases during short-term fasting and declines during obese states $(1,10-$ 15). Plasma acyl-ghrelin also increases in chronic energy-restricted

\section{Related Commentary: p. 3542}

Conflict of interest: The authors have declared that no conflict of interest exists. Copyright: (5) 2019, American Society for Clinical Investigation.

Submitted: October 4, 2018; Accepted: June 11, 2019; Published: August 19, 2019.

Reference information: J Clin Invest. 2019;129(9):3909-3923.

https://doi.org/10.1172/JCl125332. states in rodents, although in humans, prolonged fasting results in a decline in plasma acyl-ghrelin $(13,15-17)$. Plasma acyl-ghrelin levels also are dynamically affected by feeding status, with levels rising preprandially and falling after a meal $(15,18)$. These changes in plasma acyl-ghrelin suggest prominent actions during conditions of energy deficiency.

Acyl-ghrelin administration increases food intake, body weight gain, and blood glucose $(1,2,11,19)$. Based on the early characterization of those functions, blockade of acyl-ghrelin action was predicted to limit food intake, body weight gain, adiposity, and hedonic eating behaviors and also to lower blood glucose $(1,2,11,12,18,20,21)$. However, the initial hope for GHSR antagonism as a therapy for obesity and/or type 2 diabetes mellitus was tempered by results from genetic mouse models lacking ghrelin, GHSR, or GOAT, which do not exhibit marked reductions in food intake or blood glucose when food availability is plentiful and do not fully resist the development of diet-induced obesity (DIO) (22-29). Furthermore, exogenous acyl-ghrelin's orexigenic effects are less potent or absent in DIO mice, suggesting resistance to acyl-ghrelin action (ghrelin resistance) in obesity (30-34). Even so, there is mounting evidence for the involvement of endogenous acyl-ghrelin in maintaining blood glucose during energyrestricted states, at least in part through stimulation of growth hormone $(\mathrm{GH})$ secretion $(16,17,25,35,36)$, and its contributions to hyperphagia and hyperglycemia in some diabetes models (37-40). 
The inconsistent ability of endogenous ghrelin to effectively function during energy-restricted states, but not in DIO, suggests the possible existence of a regulatory molecule that may limit ghrelin action when food and nutrients are plentiful, likely as a natural adaptation to constraining continued increases in food intake and blood glucose.

Recently, the liver- and small intestine-derived peptide liverenriched antimicrobial peptide-2 (LEAP2) was reported to act as an endogenous antagonist of GHSR (41). LEAP2 was first isolated in 2003 from human blood (42) and named due to its strong homology to other endogenous antimicrobial peptides despite its weak anti-bacterial and anti-yeast properties (42). LEAP2 mRNA is expressed predominantly in liver, followed by kidney, jejunum, duodenum, stomach, and heart in humans (41-43). LEAP2 is synthesized as a 77-amino acid prohormone in humans (76 amino acids in mice) (42) that subsequently is processed to its mature form consisting of 40 amino acids with 2 disulfide bridges spanning 4 highly conserved cysteine residues $(41,44)$. The mature LEAP2 sequence is identical in mice and humans. In an attempt to identify novel secreted peptide metabolic regulators following vertical sleeve gastrectomy (VSG) surgery in mice, the Kaplan lab identified LEAP2 mRNA expression as being increased in the stomach remnant and decreased in the duodenum following VSG surgery (41). They proceeded to test the activity of LEAP2 against $168 \mathrm{G}$ protein-coupled receptors, demonstrating potent GHSR antagonist activity (41). In cell expression systems, LEAP2 dosedependently prevented acyl-ghrelin-induced increases of cytosolic calcium and $\beta$-arrestin recruitment (41). Corroborating these in vitro findings, LEAP2 administration dose dependently blocked the effects of administered acyl-ghrelin to induce food intake and GH secretion in mice (41). Viral-mediated LEAP2 overexpression phenocopied the acyl-ghrelin deficiency-associated life-threatening hypoglycemia reported in ghrelin-knockout, GOAT-knockout, ghrelin cell-ablated, and ghrelin secretion-defective mouse models submitted to a week-long $60 \%$ energy restriction protocol modeling starvation $(13,16,17,25,35)$. Suppressing endogenous LEAP2 function in mice with neutralizing antibodies was shown to boost fasting-induced increases in $\mathrm{GH}$ release, presumably by enhancing endogenous acyl-ghrelin action (41). Furthermore, plasma LEAP2, measured in lean mice using a newly developed LEAP2 sandwich ELISA assay, fell after a 24-hour fast and then rose by 1 hour after refeeding, in a pattern opposite of that of plasma total ghrelin (acyl-ghrelin + desacyl-ghrelin) (41). Plasma LEAP2 was not assessed in other metabolic conditions, nor was it determined in humans.

Here, we tested the hypothesis that LEAP2, similar to acylghrelin, represents a metabolic hormone that is regulated by body mass, feeding, and blood glucose and that works in concert with acyl-ghrelin to modulate GHSR activity as a response to those metabolic changes. We measured plasma LEAP2 in humans spanning several body mass index (BMI) categories and in both lean and obese mice, determining the influence of obesity and blood glucose. We also measured the changes in plasma LEAP2 after food intake and after 2 types of bariatric surgery in humans, upon diet-induced weight loss in mice, and in a type 1 diabetes mellitus (T1DM) mouse model. Finally, we used patch-clamp electrophysiology in mouse brain sections to test the effects of LEAP2 on spontaneous activity of GHSR-expressing hypothalamic arcuate nucleus neuropeptide Y (NPY) neurons - a well-studied target of acyl-ghrelin action $(1,21,45-48)$ - and the ability of LEAP2 to antagonize ghrelin-stimulated arcuate NPY neuronal activity.

\section{Results}

Validation of commercially available LEAP2 ELISA kit. The original report characterizing LEAP2 as an endogenous GHSR antagonist used an in-house sandwich ELISA assay to measure plasma LEAP2 in mice, demonstrating a $67 \%$ reduction following a 24 -hour fast, with partial restoration within 1 hour of refeeding (41). To extend these findings to different metabolic conditions and to humans, we first validated a commercially available LEAP2 ELISA kit from Phoenix Pharmaceuticals. This kit uses a competitive immunoassay in which a biotinylated LEAP2 peptide competes with LEAP2 peptide standard or LEAP2 peptide in the sample for binding to a polyclonal LEAP2 antibody. As the full-length peptide sequences of mature LEAP2 from humans and mice are identical (Supplemental Figure 1A; supplemental material available online with this article; https://doi.org/10.1172/JCI125332DS1), the same kit was used for both human and mouse samples. Increasing concentrations of the peptide standard dose dependently and fully competed with the biotinylated LEAP2 for binding to the LEAP2 antibody, indicating that the LEAP2 levels determined for the samples are specific to LEAP2 (Supplemental Figure 1B). Furthermore, concentrations of LEAP2 peptide from an additional source (Peptide International; catalog PLP-4405-s) estimated using the kit matched fairly well the expected concentrations, as determined from adding a known quantity of peptide to a known volume of assay buffer (Supplemental Figure 1C). Spiking mouse plasma containing endogenous LEAP2 with increasing amounts of LEAP2 peptide sourced from either Peptide International or Phoenix Pharmaceuticals caused an upward parallel shift in the concentration curves otherwise determined using the same amounts of LEAP2 peptide in assay buffer, indicating that the kit recognized both the added LEAP2 and endogenous LEAP2 (Supplemental Figure 1C). Additional validation of the kit included demonstration of a similar reduction in plasma LEAP2 concentrations upon fasting in mice, as published by the Kaplan group (ref. 41 ; see below).

Plasma LEAP2 increases in obese mice and falls after weight loss. A DIO mouse model was used to assess regulation of LEAP2 by body mass. Individually housed 4-week-old male C57BL/6N mice were provided ad libitum access to high-fat diet (HFD) or standard chow for 16 weeks. As compared with standard chow-fed mice, those fed HFD gained more body weight (Figure 1A) and developed higher fat mass and higher lean mass (Supplemental Figure 2, A and B) over the 16 weeks. Plasma LEAP2 was higher by $92 \%$ in obese mice than in lean mice (Figure 1B). In contrast, plasma acyl-ghrelin was lower by $44 \%$ in obese mice (Figure $1 \mathrm{C}$ ). We also compared plasma LEAP2 levels in each mouse to the corresponding plasma acyl-ghrelin levels, generating a plasma LEAP2/ acyl-ghrelin molar ratio. Obesity increased the plasma LEAP2/ acyl-ghrelin molar ratio by 3.3-fold (Figure 1D). Plasma LEAP2 positively correlated with fat mass (Figure 1E) and body weight (Supplemental Figure 2C). Plasma acyl-ghrelin negatively correlated with fat mass (Figure $1 \mathrm{~F}$ ) and body weight (Supplemental Figure 2D). LEAP2 mRNA expression in liver and jejunal mucosal 

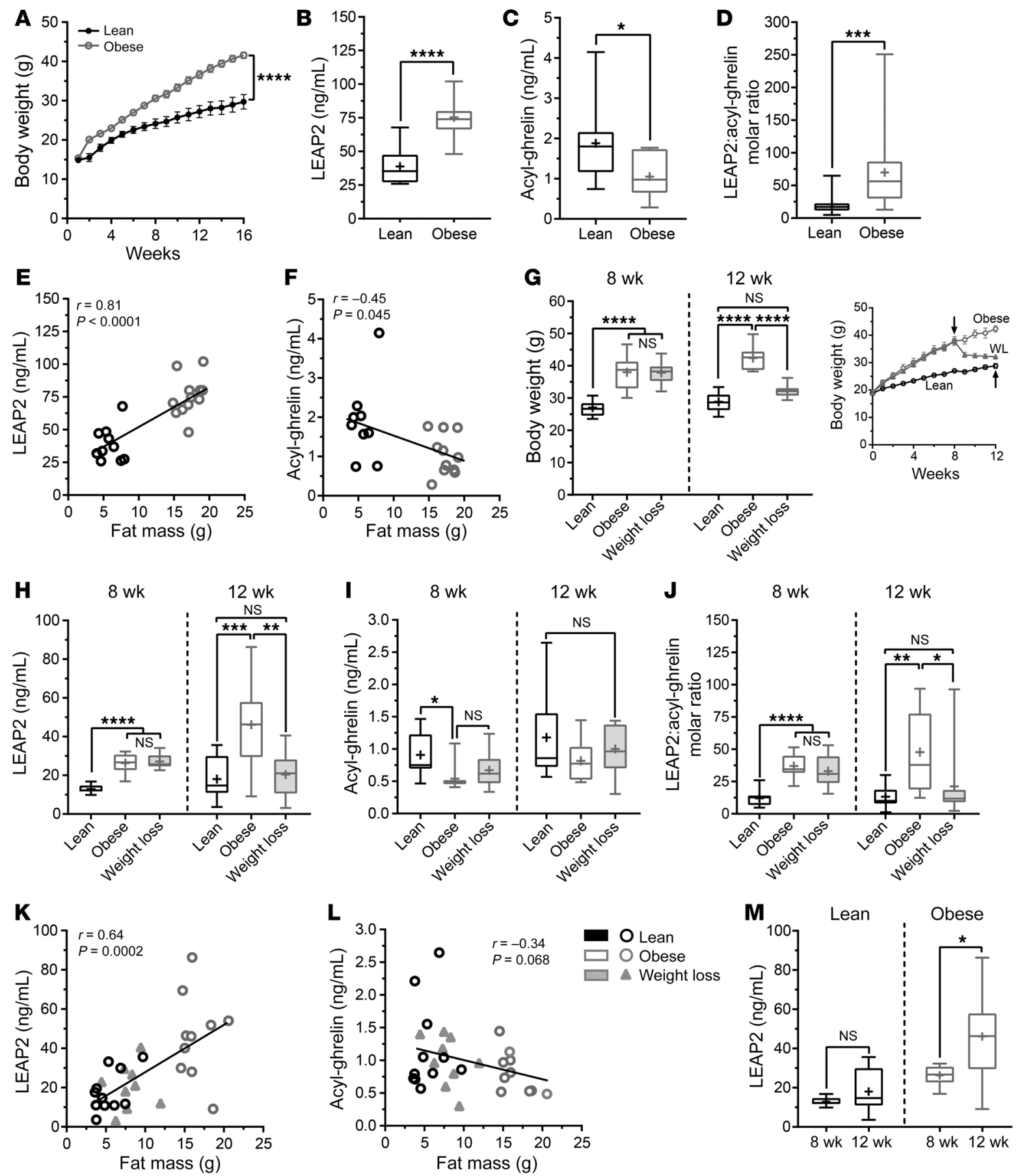

Figure 1. Responses to chronic HFD and weight loss in mice. Body weight curves of mice fed chow (lean) or HFD (obese) for 16 weeks (A). Ad libitum-fed plasma LEAP2 (B), plasma acyl-ghrelin (C), and plasma LEAP2/acyl-ghrelin molar ratio (D). Relationships of plasma LEAP2 (E) and plasma acyl-ghrelin (F) with fat mass. Body weights measured at 8 and 12 weeks in a separate cohort fed chow for 12 weeks (lean), HFD for 12 weeks (obese), or HFD for 8 weeks to induce obesity followed by chow for 4 weeks to induce weight loss (G). Inset shows body weight curves of the 3 groups throughout the study period. Arrows in the inset indicate the 8- and 12-week time points for measuring the following: plasma LEAP2 (H), plasma acyl-ghrelin (I), and plasma LEAP2/acyl-ghrelin molar ratio (J). Relationship of plasma LEAP2 (K) and plasma acyl-ghrelin (L) with fat mass at 12 weeks. Plasma LEAP2 replotted from $\mathbf{H}$ to facilitate analyzing plasma LEAP2 changes over time within the lean and obese groups (M). Data were analyzed by 2-way repeated measures ANOVA followed by Šidák's post hoc test (A), Student's unpaired $t$ test (B-D and $\mathbf{M})$, Pearson's correlation coefficient $(r)$ (E, F, K, and $\mathbf{L}), 1$-way ANOVA followed by Šidák's post hoc test (G-I), or 1-way ANOVA on ranks with post-hoc Dunn's test (J). $n=10-12(\mathbf{A}-\mathbf{F}) ; n=9-11(\mathbf{G}-\mathbf{M}) .{ }^{*} P<0.05 ;{ }^{* *} P<0.01$; ${ }^{* *} P<0.001 ; * * * P<0.0001$ 
A
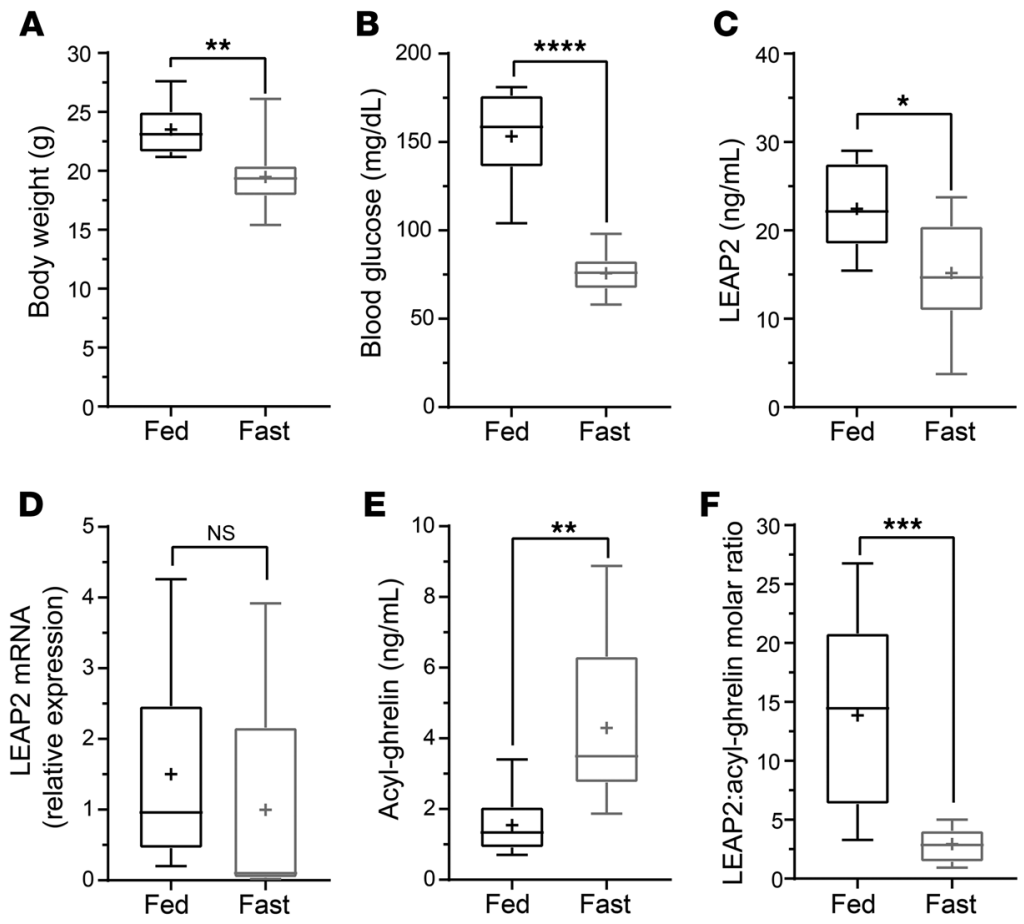

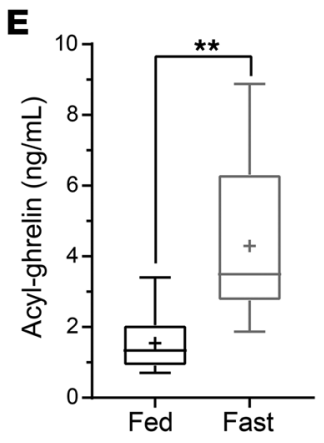

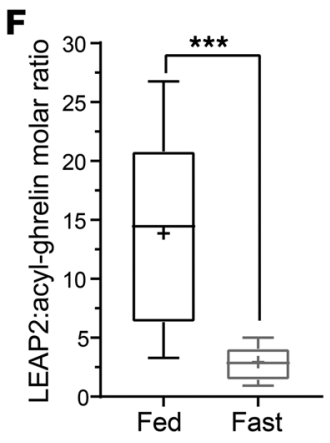

Figure 2. Responses to a 24-hour fast in lean mice. Body weight (A), blood glucose (B), plasma LEAP2 (C), liver LEAP2 mRNA expression (D), plasma acyl-ghrelin (E), and plasma LEAP2/acyl-ghrelin molar ratio (F) in lean adult mice fed standard chow ad libitum or fasted for 24 hours. Data were analyzed by Student's unpaired $t$ test. $n=10$. ${ }^{*} P<0.05$; ${ }^{* *} P<0.01$; ${ }^{* *} P<0.001$; ${ }^{* * *} P<0.0001$.

ative correlation $(P=0.068)$ with fat mass at 12 weeks (Figure 1L) and a negative correlation with body weight at both 8 weeks and 12 weeks (Supplemental Figure 3, D and F). Interestingly, plasma LEAP2 in obese mice at 12 weeks was higher than the concentrations measured in the same mice at 8 weeks, indicating that plasma LEAP2 continues to increase with further increases in body weight (Figure $1 \mathrm{M}$ ).

Overall, these data indicate that in mice, plasma LEAP2 is positively correlated with body weight and fat mass, whereas acyl-ghrelin is negatively correlated with those parameters, leading to an elevated plasma LEAP2/acyl-ghrelin molar ratio in obesity. Furthermore, diet-induced weight loss can cells of obese mice did not differ from that in lean mice (Supplemental Figure 2E), suggesting that the higher plasma LEAP2 in obese mice is likely not due to transcriptional upregulation.

Next, we tested to determine whether the increased plasma LEAP2 and decreased acyl-ghrelin in DIO can be reversed by weight loss. A separate cohort of individually housed 4- to 5-weekold male C57BL/6N mice were fed HFD for 8 weeks to induce obesity and then either allowed to remain on HFD for 4 more weeks or switched back to standard chow for 4 weeks to induce weight loss. After switching to standard chow, the obese mice lost significant body weight, so that their weights were statistically indistinguishable from the body weights of lean mice maintained on chow for 12 weeks (Figure 1G). The body weight loss was accompanied by significant loss of fat mass (Supplemental Figure 3A), but not lean mass (Supplemental Figure 3B). Plasma LEAP2 was higher in obese mice at 8 weeks than in lean mice (Figure 1H). Plasma LEAP2 fell significantly in the weight loss group after 4 weeks on chow when compared with obese mice that were continued on HFD, and levels were statistically indistinguishable from those of lean mice maintained on chow for the full 12 weeks (Figure 1H).

Plasma acyl-ghrelin was lower in obese mice at 8 weeks than in lean mice, although it did not differ among groups when measured at 12 weeks (Figure 1I). We also analyzed the coordinate changes in plasma LEAP2 and acyl-ghrelin by calculating the plasma LEAP2/acyl-ghrelin molar ratio. At 8 weeks, obese mice exhibited a higher LEAP2/acyl-ghrelin molar ratio than lean mice (Figure 1J). Weight loss caused a fall in the LEAP2/acylghrelin molar ratio (Figure 1J). The fall in fat mass of the weightloss group was accompanied by a fall in plasma LEAP2, contributing to a positive correlation of plasma LEAP2 with fat mass at 12 weeks (Figure 1K). Plasma LEAP2 also was positively correlated with body weight at both 8 weeks and 12 weeks (Supplemental Figure 3, $\mathrm{C}$ and E). Plasma acyl-ghrelin showed a trend for a negreverse obesity-associated increases in plasma LEAP2 and the plasma LEAP2/acyl-ghrelin molar ratio.

Plasma LEAP2 falls with fasting in mice. Next, we assessed the impact of fasting on plasma LEAP2 in mice. We measured plasma LEAP2 and acyl-ghrelin in 9- to 13-week-old male C57BL/6N mice that were either fed ad libitum or fasted for 24 hours. Fasted mice had lower body weights (Figure 2A) and blood glucose (Figure 2B). They also had lower plasma LEAP2 (Figure 2C). Liver LEAP2 mRNA was unchanged (Figure 2D). However, as noted previously $(11,13,49)$, plasma acyl-ghrelin was higher in fasted mice (Figure 2E). These coordinated changes in plasma LEAP2 and acylghrelin, which were similar to those reported by the Kaplan lab (41), shifted the plasma LEAP2/acyl-ghrelin molar ratio to a much lower level in fasted mice (Figure 2F). Thus, these data indicate that in mice, fasting, similarly to diet-induced weight loss, reduces plasma LEAP2 and increases plasma acyl-ghrelin, thus lowering the plasma LEAP2/acyl-ghrelin molar ratio.

Plasma LEAP2 increases in response to oral glucose administration in mice. Since plasma acyl-ghrelin is negatively regulated by oral glucose and blood glucose $(11,14,50-52)$, we tested to determine whether an acute increase in blood glucose due to glucose gavage leads to an increase in plasma LEAP2 and LEAP2/acylghrelin molar ratio. Eight- to twelve-week-old male C57BL/6N mice fasted 24 hours received $2 \mathrm{~g} / \mathrm{kg}$ glucose or the same volume of water by gavage, and blood samples were collected after 1 hour. Two weeks later, the mice underwent the same procedure, again receiving water or $2 \mathrm{~g} / \mathrm{kg}$ glucose in a crossover fashion. Oral glucose increased blood glucose (Figure 3A) and plasma LEAP2 (Figure 3B) and decreased plasma acyl-ghrelin (Figure 3C). Plasma LEAP2/acyl-ghrelin molar ratio increased by 2 -fold (Figure 3D). Blood glucose positively correlated with plasma LEAP2 (Figure 3E) and negatively correlated with plasma acyl-ghrelin (Figure 3F). Overall, these data indicate that in 
A

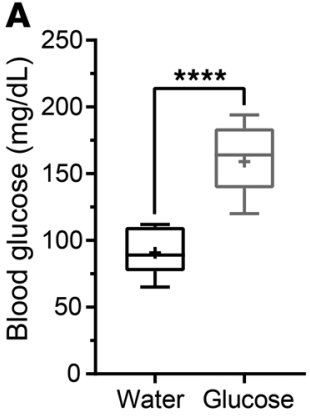

$\mathbf{F}$

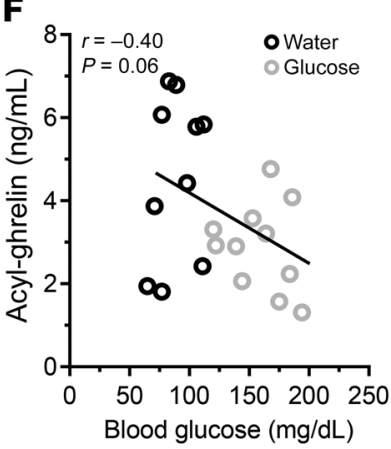

B

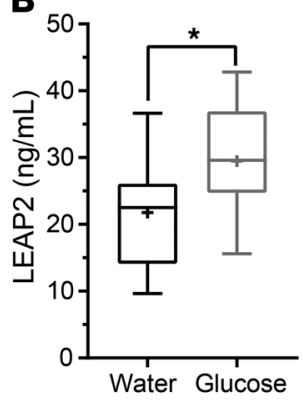

C

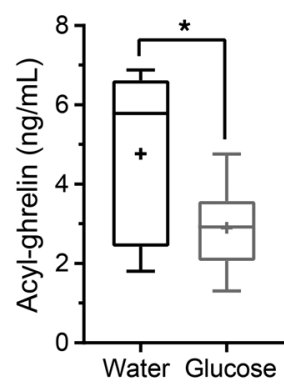

D

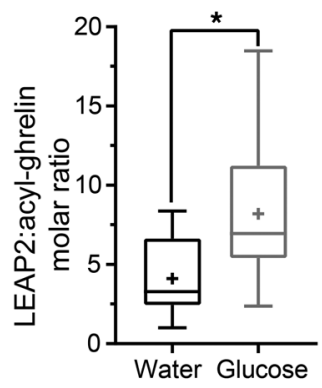

E

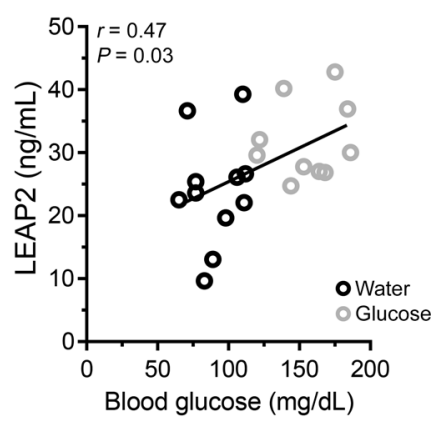

G

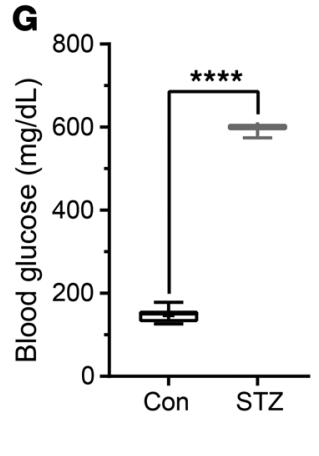

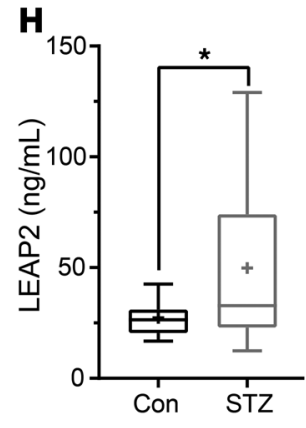

I

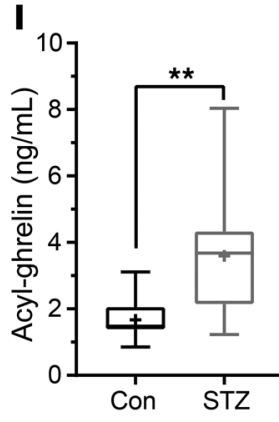

J

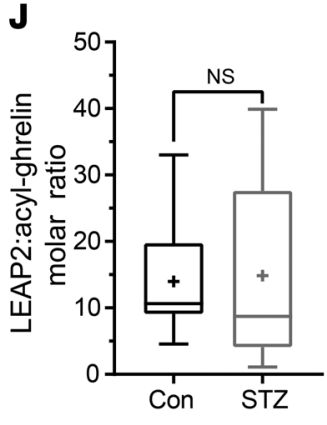

Figure 3. Responses to oral glucose administration and to induction of hyperglycemia using STZ model of T1DM in mice. Blood glucose (A), plasma LEAP2 (B), plasma acyl-ghrelin (C), and plasma LEAP2/acyl-ghrelin molar ratio (D) in mice 1 hour after oral administration of either water or 2 g/kg glucose following a 24-hour fast. Relationship of plasma LEAP2 (E) and plasma acyl-ghrelin (F) to blood glucose in those mice. Blood glucose (G), plasma LEAP2 (H), plasma acyl-ghrelin (I), and plasma LEAP2/acyl-ghrelin molar ratio (J) in mice 6 days after treatment with STZ or vehicle. Con, control. Data were analyzed by Student's unpaired $t$ test $(\mathbf{A}-\mathbf{D}$ and $\mathbf{G}-\mathbf{J})$ and Pearson's correlation coefficient $(r)(\mathbf{E}$ and $\mathbf{F}) . n=10-12$. ${ }^{*} P<0.05 ;{ }^{*} P<0.01 ;{ }^{* * *} P<0.0001$.

mice, oral glucose administration and/or the ensuing increase in blood glucose raises plasma LEAP2 and lowers plasma acylghrelin, elevating the plasma LEAP2/acyl-ghrelin molar ratio.

Plasma LEAP2 is higher in mouse T1DM model. Next, we modeled T1DM by administering streptozotocin (STZ) $(150 \mathrm{mg} / \mathrm{kg}$ body weight, i.p.) to 8 - to 10 -week-old male C57BL/6N mice. Six days later, body weight was lower (Supplemental Figure 4A) and blood glucose was higher (Figure 3G) in STZ-treated mice versus vehicle-treated mice. Plasma LEAP2 (Figure 3H) was higher in diabetic mice, as was plasma acyl-ghrelin (Figure 3I), which is in line with several previous studies $(14,38-40,53,54)$. The parallel STZ-induced increases in plasma LEAP2 and acyl-ghrelin translated to an unaltered mean plasma LEAP2/acyl-ghrelin molar ratio (Figure 3J). Liver LEAP2 mRNA expression was unaltered by STZ treatment (Supplemental Figure 4B). Thus, hyperglycemia as induced in the STZ T1DM mouse model raises plasma LEAP2, but as it does not reciprocally lower plasma acyl-ghrelin, the plasma LEAP2/acyl-ghrelin molar ratio remains unchanged.

Obesity is associated with higher plasma LEAP2 and lower plasma acyl-ghrelin in humans. Next, we assessed plasma LEAP2 and acyl-ghrelin in a cohort of adults of both sexes and spanning several BMI categories (cohort 1: $n=90$ : lean $[n=30$, BMI $\leq 25 \mathrm{~kg}$ / $\left.\mathrm{m}^{2}\right]$, overweight $\left[n=33, \mathrm{BMI}>25\right.$ to $\left.30 \mathrm{~kg} / \mathrm{m}^{2}\right]$, and obese $[n=27$, BMI $>30 \mathrm{~kg} / \mathrm{m}^{2}$, including $n=9$ with BMI $\left.>40 \mathrm{~kg} / \mathrm{m}^{2}\right]$; see Supplemental Figure 5 and Supplemental Methods for more details). Fasted plasma LEAP2 concentrations (but not acyl-ghrelin) were also available from an additional $n=15$ adults with obesity (BMI >
$35 \mathrm{~kg} / \mathrm{m}^{2}$ ) to create an expanded cohort of $n=105$ adults (cohort 1Ex: lean $\left[n=30, \mathrm{BMI} \leq 25 \mathrm{~kg} / \mathrm{m}^{2}\right]$, overweight $[n=33, \mathrm{BMI}>25$ to $30 \mathrm{~kg} / \mathrm{m}^{2}$ ], and obese [including $n=21, \mathrm{BMI}>30$ to $40 \mathrm{~kg} / \mathrm{m}^{2}$ and $\left.n=21, \mathrm{BMI}>40 \mathrm{~kg} / \mathrm{m}^{2}\right]$ ).

Fasted plasma LEAP2 was greater, fasted plasma acyl-ghrelin was lower, and fasted plasma LEAP2/acyl-ghrelin molar ratio was greater with greater BMI (Figure 4, A-C). Fasted plasma LEAP2 positively correlated with several clinical parameters associated with adverse metabolic consequences of obesity, including BMI, percentage of body fat, fasting plasma glucose, homeostatic model assessment of insulin resistance (HOMA-IR), fasting serum triglycerides, visceral adipose tissue (AT) volume (VAT), VAT/subcutaneous AT volume (SCAT) ratio, and intrahepatocellular lipid (IHCL) content, but not with SCAT (Figure 4, D-L). Relationships of fasted plasma acyl-ghrelin and fasted plasma LEAP2/acyl-ghrelin molar ratio with these parameters were also assessed (see Supplemental Figures 6 and 7, where these correlations appear alongside the just-discussed correlations with fasted plasma LEAP2, for comparison). As a result of lower plasma acyl-ghrelin in humans with obesity, fasted plasma LEAP2/acyl-ghrelin molar ratio had even stronger positive correlations with BMI, percentage of body fat, and HOMA-IR than did plasma LEAP2 alone (Supplemental Figure 6). Plasma LEAP2/acylghrelin molar ratio was positively correlated with plasma glucose $(P$ $=0.086$ ), but not with serum triglycerides (Supplemental Figure 6). Unlike LEAP2, there were no significant correlations between fasted plasma acyl-ghrelin or fasted plasma LEAP2/acyl-ghrelin molar ratio with VAT, SCAT, VAT/SCAT ratio, or IHCL (Supplemental 
A

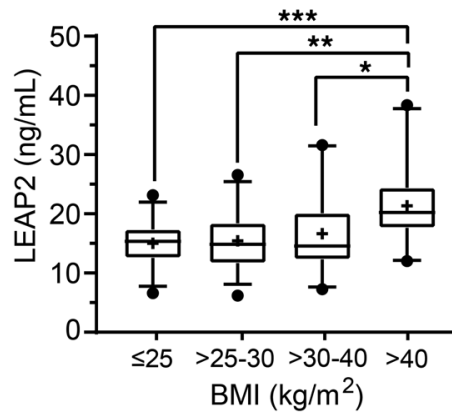

F

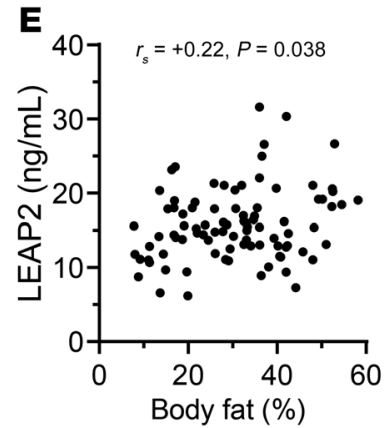

I

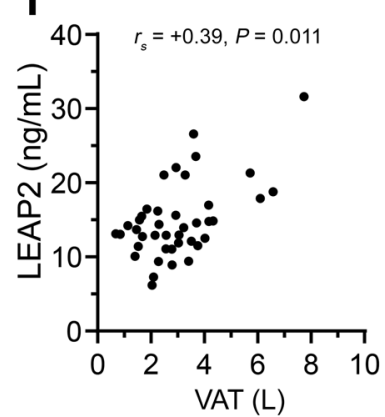

B

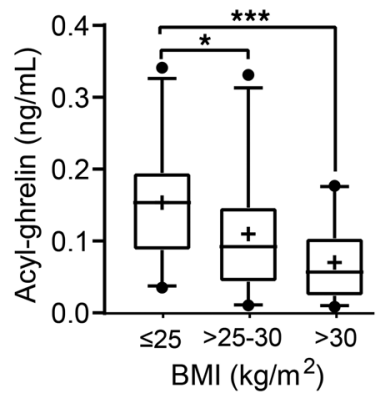

C

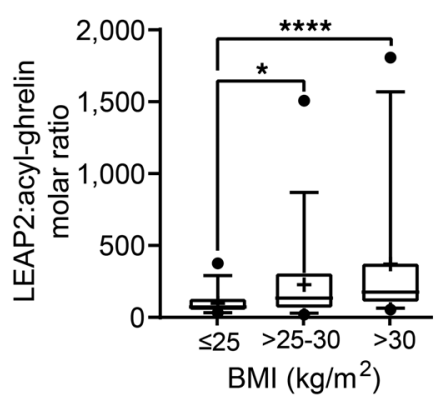

G

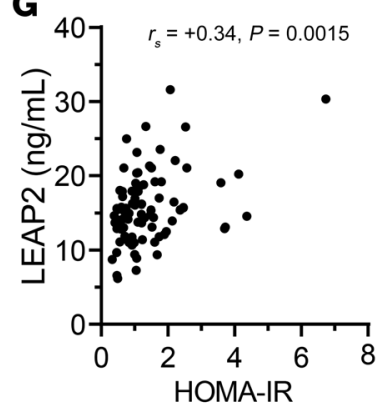

K
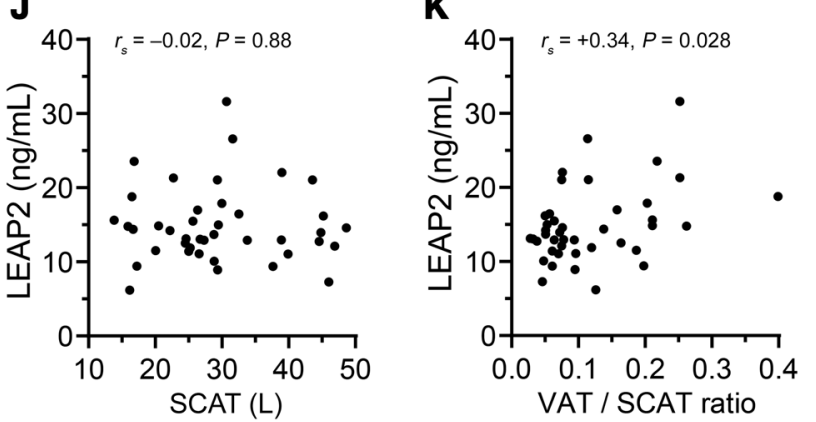

D

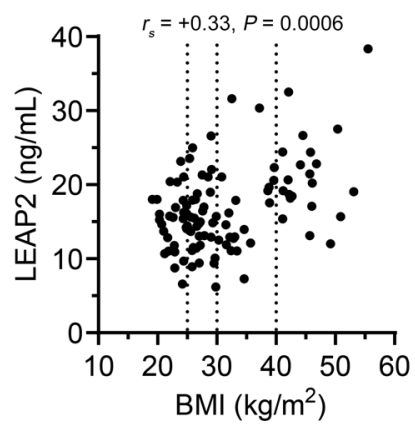

H

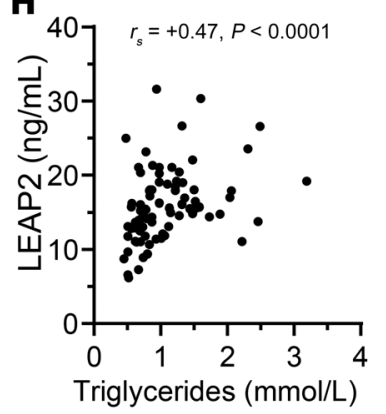

L

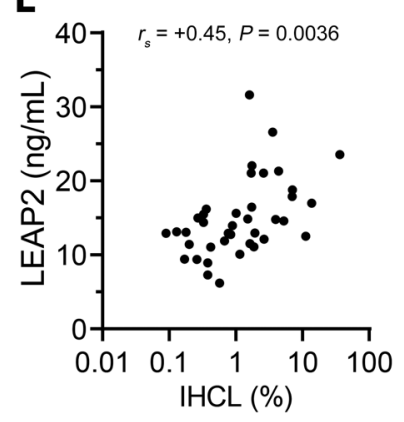

Figure 4. Changes associated with obesity in fasted humans. Fasted concentrations of plasma LEAP2 (A), plasma acyl-ghrelin (B; as assessed using 2-site University of Virginia acy-ghrelin ELISA), and plasma LEAP2/acyl-ghrelin molar ratio (C) in a cohort of adults across BMI categories. Relationships of fasted plasma LEAP2 with BMI (D), percentage of body fat (E), plasma glucose (F), HOMA-IR (G), serum triglycerides (H), VAT (I), SCAT (J), VAT/SCAT ratio (K), and percentage of IHCL (L). All data in this figure are from cohort 1, except those depicted in $\mathbf{A}$ and $\mathbf{D}$, which are from cohort 1 Ex. Data in panel $\mathbf{L}$ are represented in semi-logarithmic scale $\left(\log _{10} x\right.$ axis). Data were analyzed by 1-way ANOVA on ranks with post hoc Dunn's test (A-C) or Spearman's correlation coefficient $\left(r_{\mathrm{s}}\right)(\mathbf{D}-\mathbf{L}) . n=21-33(\mathbf{A}-\mathbf{C}) ; n=105$ (D); $n=90$ (E and F); $n=84$ (G and $\left.\mathbf{H}\right) ; n=41$ (I-K); and $n=40$ (L). ${ }^{*} P<0.05$; ${ }^{* *} P<0.01$; ${ }^{* *} P<0.001$; ${ }^{* * *} P<0.0001$.

Figure 7). Furthermore, there were no significant correlations of fasted plasma LEAP2, acyl-ghrelin, or LEAP2/acyl-ghrelin molar ratio with tibialis anterior or soleus intramyocellular lipid (IMCL) content (data not shown).

Overall, these data indicate that similarly to obesity in mice, obesity in humans is associated with higher plasma LEAP2, lower plasma acyl-ghrelin, and, in turn, a higher plasma LEAP2/acyl-ghrelin molar ratio. Furthermore, plasma LEAP2 is positively correlated with several adverse metabolic parameters associated with obesity, including BMI, percentage of body fat, HOMA-IR, fasting plasma glucose and serum triglycerides, VAT volume, and IHCL content.

Food intake increases plasma LEAP2 in humans with obesity. Next, we independently measured plasma LEAP2 and acyl-ghrelin in samples collected from a cohort of women with obesity $(n=20$, BMI $>35 \mathrm{~kg} / \mathrm{m}^{2}$ ) and age-matched normal-weight women (control; $n=12, \mathrm{BMI}<25 \mathrm{~kg} / \mathrm{m}^{2}$ ) to highlight the impact of food intake on plasma LEAP2 and acyl-ghrelin (cohort 2; see Supplemental Figure 8 and Supplemental Methods for more details). These women were in a study designed primarily to assess impact of obesity on brain activation in response to food images; the imaging part of that study is published (55). Plasma LEAP2 and acyl-ghrelin were measured in these women after an overnight fast ( 0 hours) and again postprandially ( 1.5 hours after the start of a standard $337 \mathrm{kcal}$ meal, which was consumed during a 1-hour allocated meal time).

Similarly to those in cohort 1 , the cohort 2 women with obesity had higher plasma LEAP2 compared with normal-weight women (Figure 5A). Indeed, fasted plasma LEAP2 concentrations positively correlated with BMI (Figure $5 \mathrm{~B}$ ). Meal ingestion did not change plasma LEAP2 of normal-weight women, but did increase plasma LEAP2 in women with obesity ( $P=0.08$; Figure $5 \mathrm{~A})$. This 
A
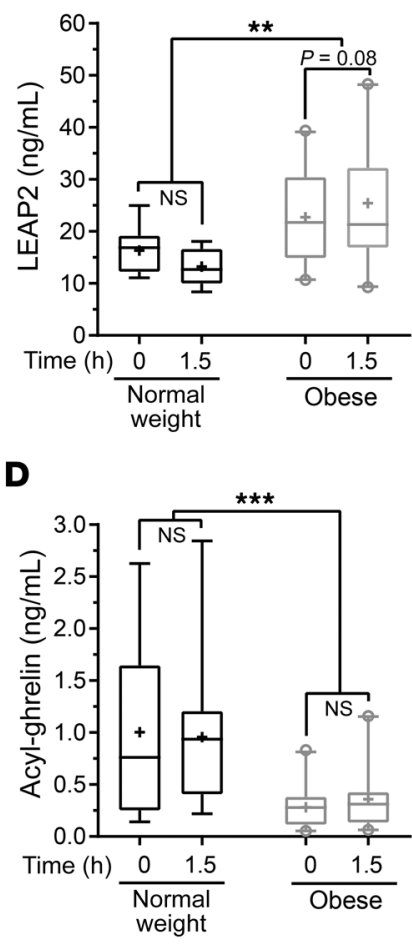

G
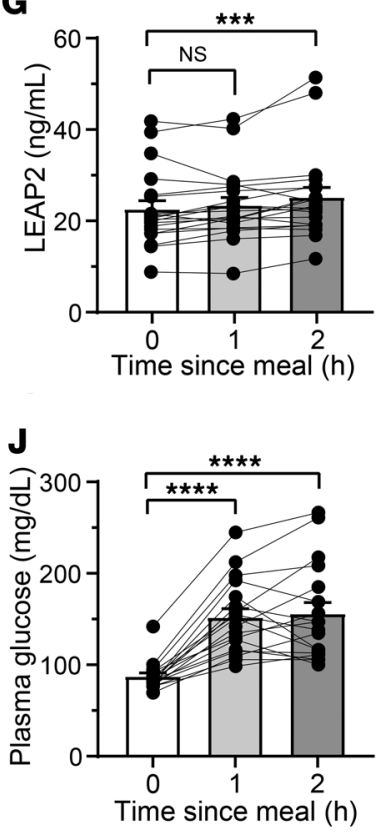

B

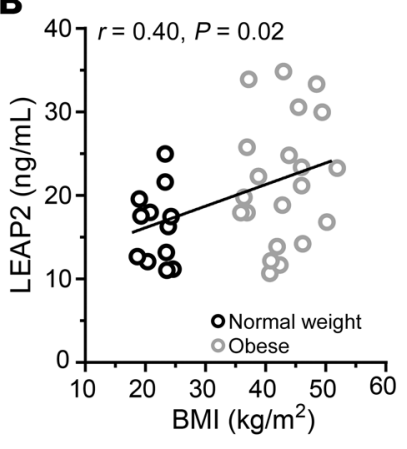

E

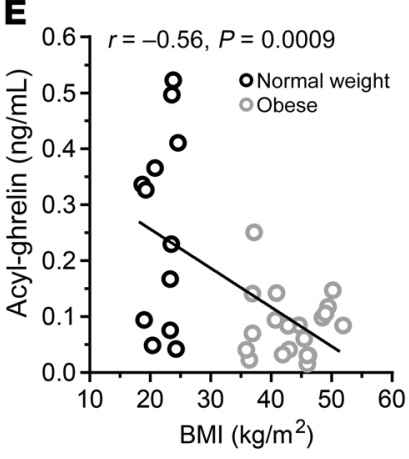

H

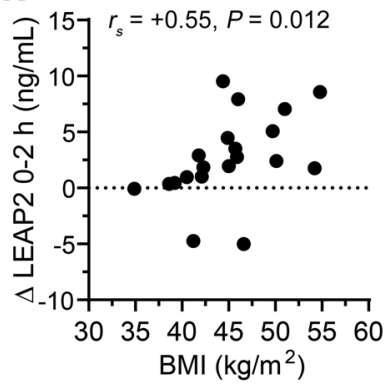

K

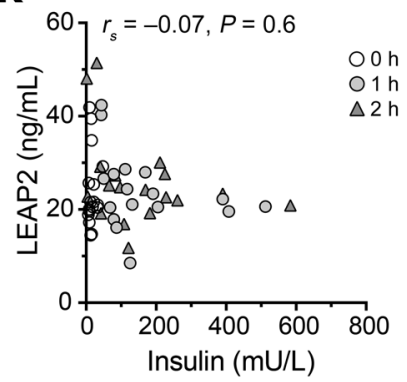

C

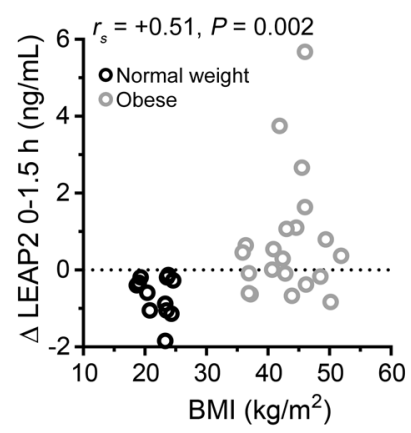

$\mathbf{F}$

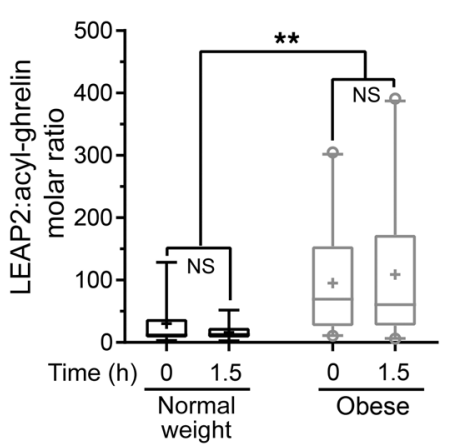

I

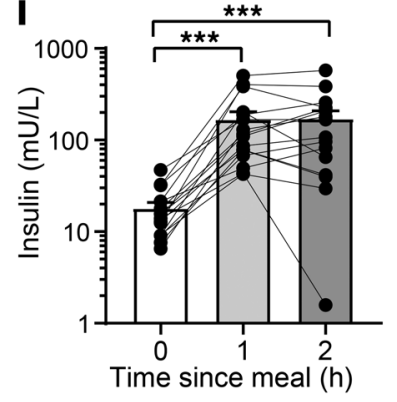

L

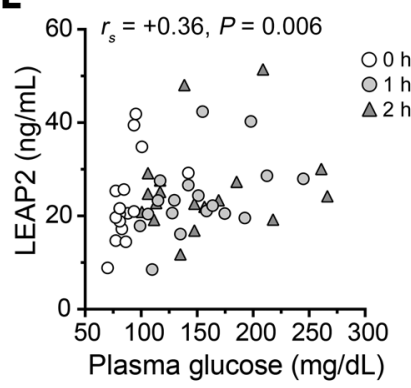

Figure 5. Changes associated with food intake in humans. Plasma LEAP2 (A) at 0 hours (after an overnight fast) and 1.5 hours (light gray) after eating a $377 \mathrm{kcal}$ standard meal in normal weight women $\left(\mathrm{BMI}<25 \mathrm{~kg} / \mathrm{m}^{2}\right)$ and in women with obesity (BMI $>35 \mathrm{~kg} /$ $\mathrm{m}^{2}$ ) (cohort 2). (B) Relationship of fasted plasma LEAP2 (0 hours) with BMI in cohort 2. (C) Relationship of the postprandial change in plasma LEAP2 (from baseline to 1.5 hours after the start of feeding $\triangle$ LEAP2 0 to 1.5 hours) with BMI in cohort 2. (D) Corresponding plasma acyl-ghrelin concentrations (Millipore assay) of cohort 2. (E) Relationship of fasted plasma acyl-ghrelin (0 hours) with BMI in cohort 2. (F) Changes to LEAP2/ acyl-ghrelin molar ratio in cohort 2. (C) Plasma LEAP2 at 0 hours, 1 hour (light gray), and 2 hours (dark gray) after consuming a $600 \mathrm{kcal}$ liquid meal in adults with obesity (BMI > $35 \mathrm{~kg} / \mathrm{m}^{2}$ ) (cohort 3). (H) Relationship of the postprandial change in plasma LEAP2 (from baseline to 2 hours after food intake; $\triangle$ LEAP2 0 to 2 hours) with $B M I$ in cohort 3 . Corresponding serum insulin (I), and plasma glucose (J) concentrations of cohort 3. Relationships of plasma LEAP2 with serum insulin (K) and plasma glucose $(\mathrm{L})$ at baseline $(0$ hours), 1 hour, and 2 hours after consumption of the diet in cohort 3. Data in panel I are represented in semi-logarithmic scale $\left(\log _{10} y\right.$ axis). Data were analyzed by 2-way repeated measures ANOVA followed by Šidák's post hoc test (A, D, and F), Pearson's correlation ( $r$ ) (B and E), Spearman's correlation $\left(r_{s}\right)(\mathbf{C}$, $\mathbf{H}, \mathbf{K}$, and $\mathbf{L}$ ), or 1-way repeated measures ANOVA on ranks with post hoc Dunn's test (G, I, and J). $n$ $=12$ for normal weight women; $n=$ 20 for women with obesity (A-F); $n=20$ (G-L). Data in $\mathbf{G}, \mathbf{I}$, and $\mathbf{J}$ are represented as mean \pm SEM. ${ }^{* *} P<$ $0.01 ;{ }^{* *} P<0.001 ;{ }^{* * *} P<0.0001$. postprandial change in plasma LEAP2 ( $\triangle$ LEAP2 0 to 1.5 hours) positively correlated with BMI (Figure 5C). Also, similarly to what occurred in cohort 1 (and as expected; refs. 56-58), plasma acylghrelin of cohort 2 women with obesity was lower than that of normal-weight women (Figure 5D), with plasma acyl-ghrelin concentrations negatively correlating with BMI (Figure 5E). However, plasma acyl-ghrelin did not change with food intake in either the normal-weight women or women with obesity (Figure 5D), in contrast to what occurred in other clinical studies $(18,49,56)$. Finally, just as observed in cohort 1, plasma LEAP2/acyl-ghrelin molar ratio was greater in cohort 2 women with obesity as compared with normal-weight women (Figure 5F). However, these plasma LEAP2/acyl-ghrelin molar ratios did not change postprandially in the cohort 2 groups (Figure 5F).

We also examined the effect of food intake in a separate cohort of adults with obesity, including many considering Roux-en-Y gastric bypass (RYGB) surgery (cohort 3, see Supplemental Figure 9 and Supplemental Methods for more details; notably, plasma acyl-ghrelin was unavailable for cohort 3). After ingestion of a $600 \mathrm{kcal}$ liquid meal, plasma LEAP 2 was higher by 
A

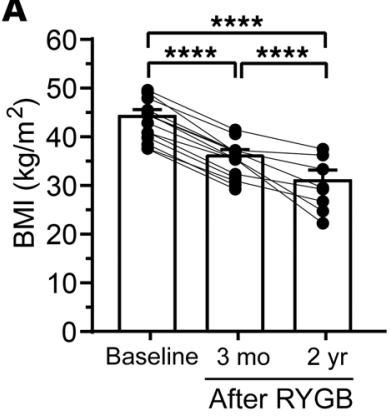

D

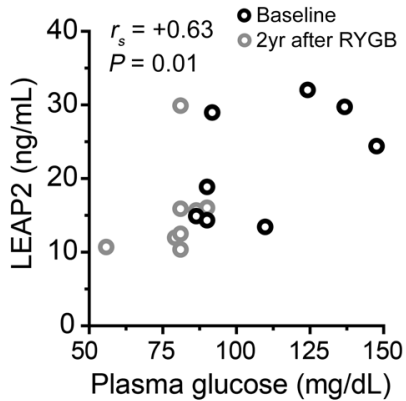

G

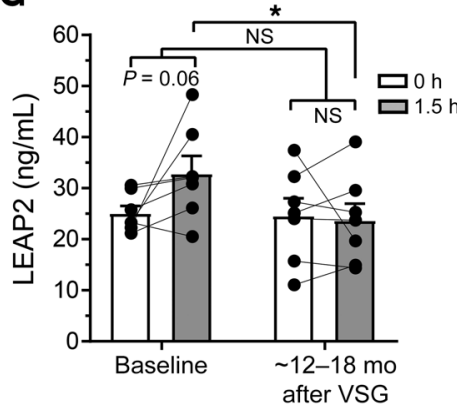

B

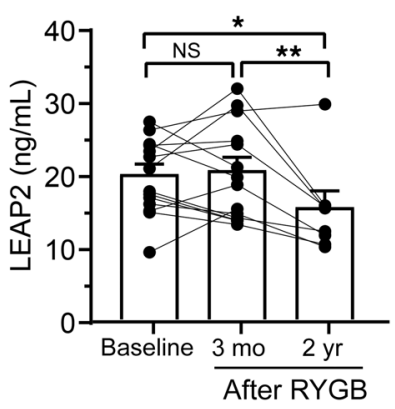

$\mathbf{E}$

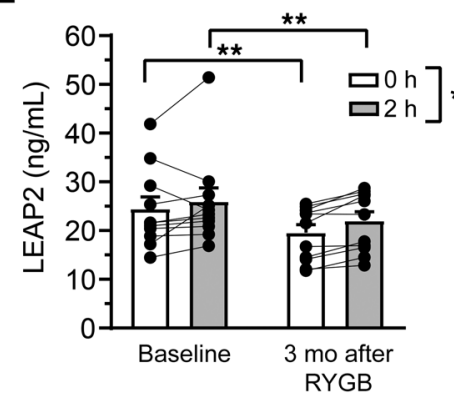

H

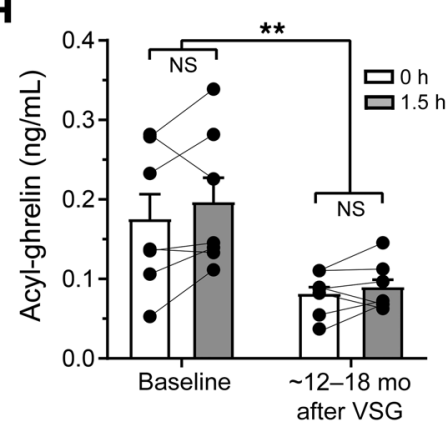

C

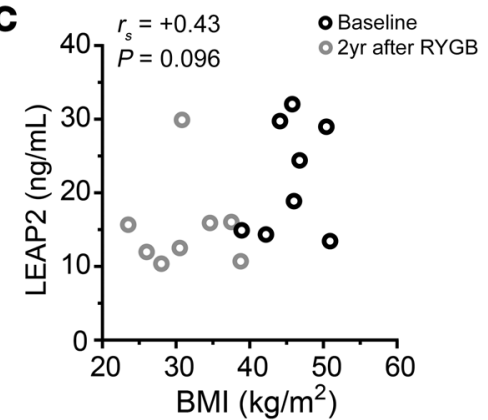

$\mathbf{F}$

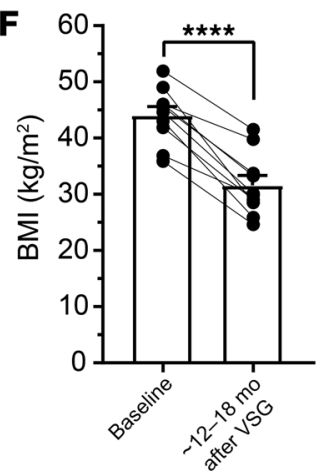

\section{I}

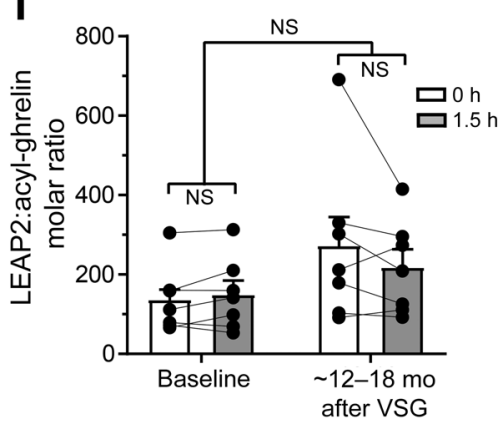

Figure 6. Effects of RYGB and VSG in humans with obesity. BMI (A) and fasted plasma LEAP2 (B) at baseline and at approximately 3 months ( $n=14$ ) and 2 years $(n=8)$ following RYGB (cohort 3A). Relationships of fasted plasma LEAP2 with BMI (C) and plasma glucose (D) before and 2 years after RYCB surgery in cohort 3A. Plasma LEAP2 (E) in adults before (baseline) and approximately 3 months after RYCB surgery before (time 0 hours) and 2 hours after ingestion of a $600 \mathrm{kcal}$ liquid meal in cohort 3B $(n=11)$. BMI $(\mathbf{F})$ at before (baseline) and at approximately 12 to 18 months following VSG surgery (cohort 2A). Plasma LEAP2 (G), acyl-ghrelin (H) (Millipore assay), and LEAP2/acyl-ghrelin molar ratio (I) in women with obesity before (baseline) and approximately 12 to 18 months after VSG surgery before (time 0 hours) and 1.5 hours after the start of consuming a standard $337 \mathrm{kcal}$ meal following an overnight fast (cohort 2A). $n=7$ (F-I). Data were analyzed by mixed effects ANOVA, followed by Šidák's post hoc tests (A and B), Spearman's correlation $\left(r_{s}\right)(\mathbf{C}$ and $\mathbf{D}), 2$-way repeated measures ANOVA with Šidák's post-hoc test $(\mathbf{E}$ and $\mathbf{G}-\mathbf{I})$, and Students paired $t$ test $(\mathbf{F})$. Data in graphs $(\mathbf{A}, \mathbf{B}$, and $\mathbf{E}-\mathbf{I})$ are represented as mean \pm SEM. ${ }^{*} P<0.05 ;{ }^{* *} P<0.01 ;{ }^{* * *} P<0.0001$.

2 hours postprandially (Figure $5 \mathrm{G}$ ). Similarly to the 1.5 -hour postprandial correlations in cohort 2 (Figure $5 \mathrm{C}$ ), the 2-hour postprandial change in plasma LEAP2 ( $\triangle$ LEAP2 0 to 2 hours) in cohort 3 also positively correlated with BMI (Figure $5 \mathrm{H}$ ). Serum insulin and plasma glucose were higher by 1 hour postprandially (Figure 5 , I and J). Plasma LEAP2 measured at baseline (0 hours) and at 1 hour and 2 hours postprandially did not correlate with serum insulin (Figure $5 \mathrm{~K}$ ), but did positively correlate with plasma glucose (Figure 5L).

Thus, plasma LEAP2 increases to a greater degree postprandially in individuals with higher BMI. Also, similarly to what occurred in mice, plasma LEAP2 is positively correlated with blood glucose in humans.
RYGB and VSG surgery reduce plasma LEAP2 in humans. We determined changes in plasma LEAP2 following RYGB in 2 cohort 3 subsets and following VSG in a cohort 2 subset by comparing the baseline data (Figure 5) to postbariatric surgery data for those participants in whom both data sets were available.

Cohort 3A had overnight-fasted plasma LEAP2 measurements both before and at approximately 3 months after RYGB surgery ( $n=14$ from cohort 3; see Supplemental Figure 9 and Supplemental Methods for more details), while $n=8$ of these patients were also studied at approximately 2 years after RYGB. BMI fell from baseline at both post-RYGB time points, with a $31.5 \% \pm 3.7 \%$ weight loss by 2 years (Figure 6A). Fasted plasma LEAP2 was significantly lower at 2 years after RYGB, but not at 3 months after 

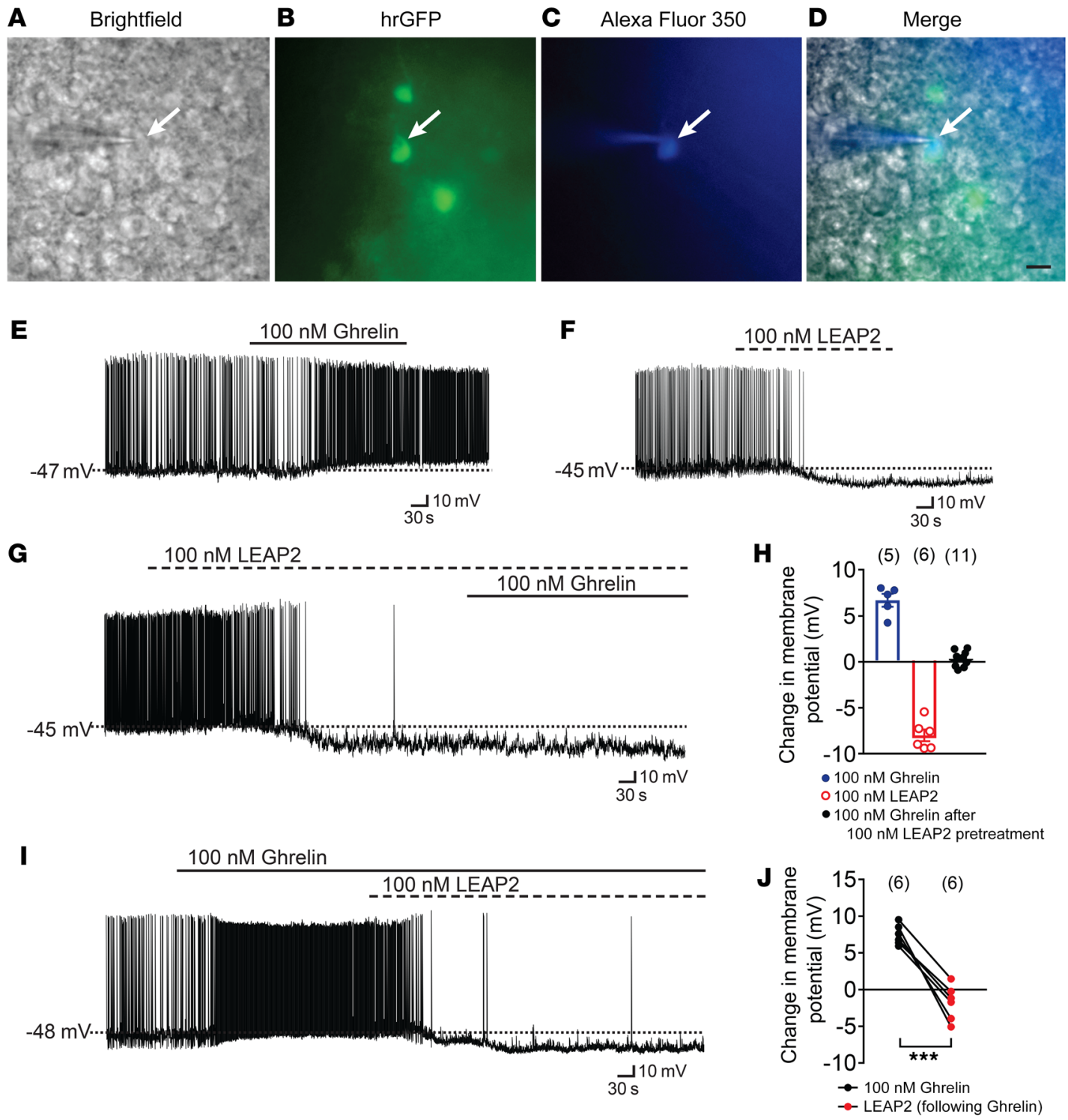

Figure 7. LEAP2 effects on arcuate hypothalamic NPY neuronal activity in mice. (A) Brightfield illumination of a glass pipette patched onto a representative arcuate NPY-hrGFP neuron in a coronal brain section from an NPY-hrGFP mouse. (B) hrGFP fluorescence of the same neuron. (C) Complete dialysis of the neuron with Alexa Fluor 350. (D) Merged image of the NPY neuron targeted for electrophysiological recording. Scale bar: 50 $\mu \mathrm{m}$. Arrows in A-D indicate the targeted NPY neuron. (E) A representative current-clamp record depicting the characteristic depolarization of arcuate NPY neurons by ghrelin (100 nM). (F) A representative current-clamp record showing hyperpolarization of NPY neuron by LEAP2 (100 nM). (G) A representative current-clamp trace demonstrating that ghrelin (100 nM) fails to alter the membrane potential of a NPY neuron that had previously been inhibited by LEAP2 (100 nM). (H) Scatter plot with bar illustrates the acute effects of acyl-ghrelin (100 nM, blue) and LEAP2 (100 nM, red) on the membrane potential of arcuate NPY neurons and that of ghrelin $(100 \mathrm{nM})$ on the membrane potential of arcuate NPY neurons pretreated with and in the continued presence of LEAP2 (100 nM, black). (I) Representative current-clamp trace demonstrates reversal of acyl-ghrelin-induced depolarization of arcuate NPY neurons with addition of LEAP2 (100 nM). (J) Line graph illustrates the acute effects of acyl-ghrelin (100 nM, black) and the effect of the subsequent addition of LEAP2 (100 nM, red) in the continued presence of acyl-ghrelin on the membrane potential of arcuate NPY neurons. ${ }^{* *} P<0.001$. Numbers within brackets indicate $n$ for each experiment. Data in $\mathbf{H}$ are represented as mean \pm SEM.

RYGB (Figure 6B). Analysis of the baseline data together with the 2-year post-RYGB data showed positive correlations between fasted plasma LEAP2 with BMI $(P=0.096)$ and plasma glucose $(P=0.01)$ (Figure 6, C and D).

Cohort 3B had baseline ( 5 to 6 hours after usual breakfast at home) and postprandial LEAP2 measurements both before and approximately 3 months after RYGB surgery, when mean percentage of weight loss was $18.3 \pm 2.0$ ( $n=11$ from cohort 3 ; see Sup- plemental Figure 9 and Supplemental Methods for more details). Plasma LEAP2 increased by 2 hours after consumption of the 600 kcal liquid meal, with no effect of RYGB surgery on the postprandial increase (feeding state $\times$ visit interaction, $P=0.52$; effect of feeding state, $P=0.017$ ) (Figure $6 \mathrm{E}$ ). Furthermore, both baseline and 2 hours postprandial plasma LEAP2 significantly decreased after RYGB surgery (overall effect of visit independent of feeding state, $P=0.055$ ) (Figure $6 \mathrm{E}$ ). 


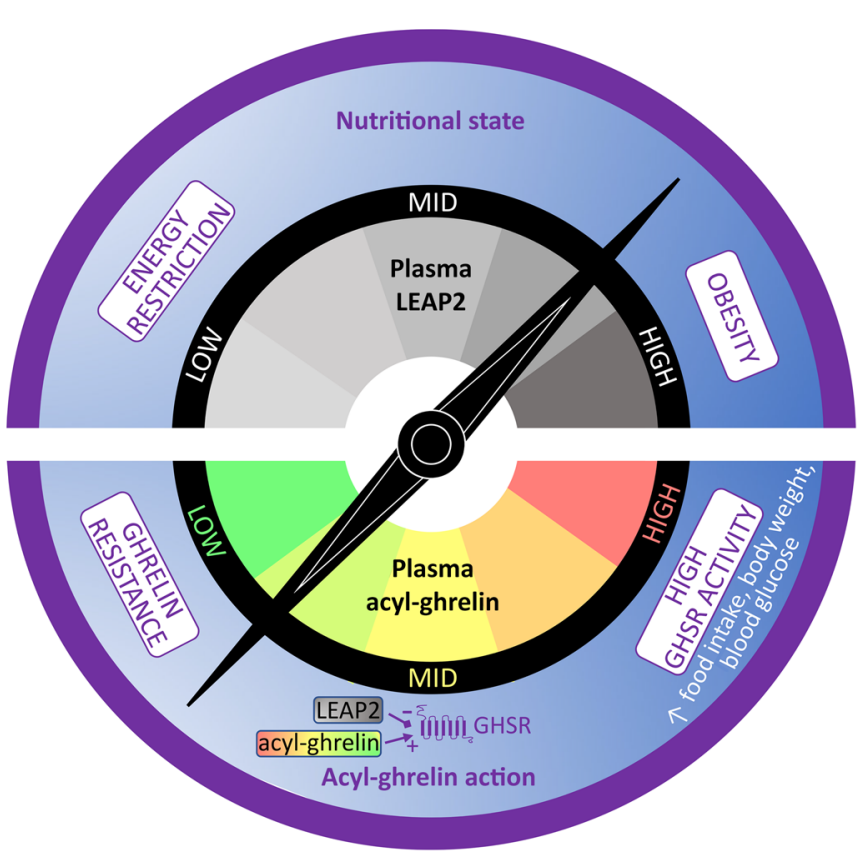

Cohort 2A had measurement of plasma LEAP2 and acylghrelin after an overnight fast ( 0 hours) and 1.5 hours after the start of a standard $337 \mathrm{kcal}$ meal, both before and approximately 12 to 18 months after VSG surgery $(n=7$, women with obesity from cohort 2; see Supplemental Figure 8 and Supplemental Methods for more details). Mean percentage of weight loss was $28.8 \% \pm$ $2.7 \%$ by approximately 12 to 18 months after VSG surgery (Figure $6 \mathrm{~F})$. Postprandial change in plasma LEAP2 by 1.5 hours after consumption of the meal varied with VSG surgery (feeding state $\times$ visit interaction $P=0.07$ ), with a statistical trend toward a postprandial increase in LEAP2 observed before VSG surgery $(P=0.06)$, but not at approximately 12 to 18 months after VSG surgery $(P=$ 0.94) (Figure 6G). Furthermore, the 1.5 hours postprandial plasma LEAP2 was significantly lower after VSG surgery when compared with before VSG surgery (Figure 6G). In contrast, both 0 hours and 1.5 hours postprandial plasma acyl-ghrelin significantly decreased approximately 12 to 18 months after surgery (overall effect of visit independent of feeding state, $P=0.009$ ), with no feeding-dependent change in acyl-ghrelin during either visit (Figure 6H). For cohort 2A, neither VSG nor consuming the standard meal affected mean plasma LEAP2/acyl-ghrelin molar ratio, nor was a feeding state $\times$ visit interaction observed (Figure 6I).

These data indicate that plasma LEAP2 decreases in humans after 2 different types of weight loss surgery. RYGB lowers fasted plasma LEAP 2 by 2 years after surgery and postprandial plasma LEAP 2 by 3 months after surgery, but does not alter the magnitude of the meal-induced increase in plasma LEAP2. VSG lowers postprandial plasma LEAP2 (as compared with the baseline postprandial state) and also prevents the meal-induced increase in plasma LEAP2. VSG also lowers plasma acyl-ghrelin regardless of meal status, leading to no overall change in plasma LEAP2/acyl-ghrelin molar ratio by approximately 12 to 18 months after VSG.

LEAP2 acts as a GHSR antagonist and inverse agonist that hyperpolarizes arcuate NPY neurons. To further characterize LEAP2 effects on GHSR action, we performed whole-cell patch-clamp recordings
Figure 8. Model of the effect of LEAP2 on acyl-ghrelin-mediated GHSR action. The hormones LEAP2 and acyl-ghrelin both bind to GHSR. Acylghrelin stimulates GHSR activity, while LEAP2 acts as a GHSR inverse agonist that blocks constitutive and acyl-ghrelin-mediated CHSR activity. A fall in plasma LEAP2 during deficient nutritional states (e.g., energy restriction and fasting) is usually associated with a coordinate rise in plasma acyl-ghrelin; these together create a permissive environment in which elevated acyl-ghrelin can most effectively act, for instance, to induce feeding, raise body weight, and/or prevent potentially life-threatening falls in blood glucose. In contrast, in states of nutritional abundance (e.g., obesity and food intake), a usual coordinated rise in plasma LEAP2 and fall in plasma acyl-ghrelin limits acyl-ghrelin's orexigenic and blood glucose-raising actions so as to minimize food intake and increases in blood glucose. A shift in the plasma LEAP2/acyl-ghrelin molar ratio to higher levels may be a key determinant of ghrelin resistance that helps limit the contribution of acyl-ghrelin to obesity-associated morbidity.

of arcuate hypothalamic NPY neurons, which highly express GHSRs and mediate some of acyl-ghrelin's orexigenic efficacy $(45,47,48$, 59-61), in brain sections prepared from NPY-hrGFP mice (Figure 7 , A-D). Similarly to previous reports $(45,46)$, application of ghrelin (100 nM) depolarized NPY neurons (change of mean membrane potential was $6.7 \pm 0.7 \mathrm{mV}$; Figure 7, $\mathrm{E}$ and $\mathrm{H}$ ). In contrast, application of LEAP2 (100 $\mathrm{nM}$ ) hyperpolarized NPY neurons (change of mean membrane potential was $-8.0 \pm 0.6 \mathrm{mV}$; Figure $7, \mathrm{~F}$ and $\mathrm{H}$ ). Addition of ghrelin (100 nM) failed to alter NPY neuron membrane potential in brain slices pretreated with LEAP2 (100 nM; change of mean membrane potential was $0.3 \pm 0.2 \mathrm{mV}$; Figure $7, \mathrm{G}$ and $\mathrm{H}$ ). Furthermore, addition of 100 nM LEAP2 reversed ghrelin-induced membrane depolarization in all ghrelin-responsive NPY neurons examined (change of mean membrane potential with ghrelin application $=7.5 \pm 0.6 \mathrm{mV}$ [Figure $7, \mathrm{I}$ and J] vs. change in mean membrane potential with addition of LEAP2 $=-9.3 \pm 1.1 \mathrm{mV}$ [Figure 7, I and J]). Notably, application of LEAP2 to ghrelin-exposed NPY neurons hyperpolarized (by $-5.1 \mathrm{mV}$ and $-4.0 \mathrm{mV}$ ) 2 of the 6 examined NPY neurons further from the starting resting membrane potential measured before the addition of ghrelin. Thus, these data suggest that LEAP2 not only acts as a powerful GHSR antagonist that can incapacitate acyl-ghrelin-induced activation of arcuate NPY neurons, but also functions as a GHSR inverse agonist that disables GHSR constitutive activity and, in so doing, hyperpolarizes NPY neurons and prevents acyl-ghrelin from activating them.

\section{Discussion}

In their seminal 2018 paper, Ge et al. (41) identified LEAP2 as an endogenous GHSR antagonist using in vitro assays. They demonstrated that LEAP2 dose dependently attenuates acyl-ghrelininduced food intake and GH secretion in mice, that LEAP2 neutralization boosts fasting-induced GH release in mice, and that LEAP2 overexpression reproduces the life-threatening hypoglycemia observed in other models of deficient acyl-ghrelin action during an energy restriction regimen modeling starvation. Furthermore, 
they demonstrated that fasting is associated with lowering of plasma LEAP2 in mice, with partial restoration of plasma LEAP2 by refeeding, in a pattern opposite of that of plasma acyl-ghrelin. Here, we report clinically relevant findings regarding changes in plasma LEAP2 in states of altered energy balance and metabolism. These include relationships with obesity and its associated adverse metabolic consequences plus effects of food intake and weight loss through energy restriction or bariatric surgery, which are complementary in both mice and humans. We have validated the commercially available LEAP2 ELISA assay kit used in our studies. Furthermore, we characterized LEAP2 actions as a GHSR inverse agonist using whole-cell patch-clamp recordings of arcuate NPY neurons within mouse brain sections.

Plasma LEAP2 was higher in obesity, positively correlating with BMI, percentage of body fat, plasma glucose, HOMA-IR, serum triglycerides, VAT, VAT/SCAT ratio, and IHCL content in humans and with fat mass and body weight in mice. These findings contrasted with those for plasma acyl-ghrelin, which was generally lower in obese states. This translated to an increase in the mean plasma LEAP2/acyl-ghrelin molar ratio in obese subjects when compared with control subjects (from 21:1 in lean mice to 70:1 in obese mice and from 28:1 in normal weight humans to 95:1 in humans with obesity). Lower BMI after bariatric surgery (RYGB or VSG) in humans or after diet-induced weight loss in mice was generally associated with lower plasma LEAP2, indicating that the rise in plasma LEAP2 linked to the development of obesity can be reversed by losing weight. In contrast, plasma LEAP2 was generally lower in fasted states than in fed states in mice, contributing to a fall in the mean plasma LEAP2/acyl-ghrelin molar ratio (from 14:1 in ad libitum-fed mice to 3:1 in fasted mice).

These results suggest that plasma LEAP2 is sensitive to body weight and feeding status and is usually (with a few exceptions noted below) regulated in a manner diametrically opposite to that of plasma acyl-ghrelin. We propose a model (Figure 8) in which the rise in plasma LEAP2 together with the fall in plasma acyl-ghrelin could be a major contributor to the ghrelin resistance observed during obese states (34). In contrast, the coordinated fall in plasma LEAP2 together with the rise in plasma acyl-ghrelin is proposed to create a permissive environment for acyl-ghrelin to potently act during energy-restricted states. Thus, we predict that the plasma LEAP2/acyl-ghrelin molar ratio could be a key determinant modulating GHSR actions in response to changes in body mass, feeding status, and blood glucose.

Ghrelin activates GHSR with an $\mathrm{EC}_{50}$ of 2.5 to $7.1 \mathrm{nM}(3,41)$, and LEAP2 acts as a GHSR antagonist with an $\mathrm{IC}_{50}$ of $6 \mathrm{nM}(41)$. Therefore, the potency of LEAP2 as a GHSR antagonist is very close to the potency of ghrelin as a GHSR agonist (41). The mean ad libitum-fed plasma LEAP2 concentration in our mouse studies (on average approximately $20 \mathrm{ng} / \mathrm{mL}$ or $4.4 \mathrm{nM}$ ) using a commercial ELISA kit was similar to the concentration measured by Ge et al. (41), who used a custom ELISA kit, and is close to the $\mathrm{IC}_{50}$ for GHSR (6 nM) (41). Therefore, we confirm the conclusion by Ge et al. (41) that LEAP2, at its physiological circulating concentrations, would be very effective as a GHSR antagonist. Such a conclusion is supported by the increases in GHSR-mediated GH secretion after immunoneutralization of endogenous LEAP2 in mice even during fasted conditions, when plasma LEAP2 is lower than during the ad libitum-fed condition (Figure 2 and ref. 41). Interestingly, the plasma LEAP2 concentrations we measured in both humans and mice are more than 1000 -fold lower than the $\mathrm{IC}_{50}$ for its originally described antimicrobial activity (approximately $5 \mu \mathrm{M}$ ), suggesting that the prominent physiological function of circulating LEAP2 is as a GHSR antagonist rather than its first characterized function as an antimicrobial peptide (42). Here, we also observed that the concentration of plasma LEAP2 in the ad libitum-fed state is more than 20-fold higher than that of plasma acyl-ghrelin in both mice and humans. Therefore, given the roughly equal potency as well as affinity of the 2 peptides for GHSR $(41,62,63)$, it is likely that in the fed state, LEAP2 serves as the dominant ligand of GHSR, prominently antagonizing acyl-ghrelin actions. In the fasted state, the fall in the LEAP2/acyl-ghrelin molar ratio likely favors a relatively higher proportion of acyl-ghrelin binding to mediate its effects through GHSR. This potentially explains why genetic deletion of endogenous ghrelin does not have pronounced metabolic effects in ad libitum-fed conditions, but does upon energy restriction (refs. 2, 16, 24, 26, 35, 64 and Figure 8).

Our electrophysiology results using mouse brain sections, as well as recent studies using heterologous cell-expression systems $(62,63)$, confirm that LEAP2 opposes acyl-ghrelin actions on GHSR. However, our findings were unlike the original discovery by $\mathrm{Ge}$ at al., which, based on experiments utilizing a $\beta$-arrestin recruitment assay in a cell line stably expressing GHSRs, described LEAP2 as a "classic" GHSR antagonist with no intrinsic activity (41). Instead, our studies demonstrating hyperpolarization of mouse arcuate NPY neurons by application of LEAP2 alone, as opposed to the expected observation of no effect on neuronal activity if LEAP2 was a "classic" antagonist, indicate that LEAP2 acts as an inverse agonist rather than simply as an antagonist of acyl-ghrelin action on GHSRs. This conclusion is further supported by a recent study that observed a $50 \%$ decrease in inositol phosphate 1 intracellular second messenger levels upon LEAP2 treatment of GHSR-expressing HEK293T cells (62).

Acyl-ghrelin stimulates food intake at least in part by engaging GHSRs expressed in orexigenic arcuate NPY/AgRP neurons (36, $45,47,61,65,66)$. However, in DIO mice, the orexigenic response to acyl-ghrelin administration is blunted or absent (30-34, 67). Also, acyl-ghrelin fails to induce arcuate c-fos immunoreactivity or increase arcuate NPY and AgRP mRNA expression in DIO mice, as occur upon acyl-ghrelin administration to chow-fed mice, suggesting the development of ghrelin resistance in obese states (19, 30-34, 68). Our results demonstrating elevated plasma LEAP2 in DIO and LEAP2 inhibition of spontaneous and acyl-ghrelininduced increases in arcuate NPY neuronal activity suggest that LEAP 2 could be an important component of the altered endocrine feedback during DIO, directly affecting GHSR function, and in particular, leading to ghrelin resistance. Future loss-of-LEAP2function genetic mouse model studies will help reveal the contribution of LEAP2 to the ghrelin resistance associated with DIO.

We also found that plasma LEAP2, similar to plasma acylghrelin, is regulated dynamically by feeding status. Plasma LEAP2 was higher in ad libitum-fed lean mice than in fasted mice, as had been noted by Ge et al. (41), whereas plasma acyl-ghrelin was lower $(1,10-15)$. Oral glucose administration had the same effect in mice 
to increase plasma LEAP2. A postprandial change in plasma LEAP2 also was observed in 2 separate human cohorts. In both cohorts, LEAP2 positively correlated with BMI, and the postprandial increase was present only in the obese group, but not the normal weight group. Thus, the higher the BMI, the more plasma LEAP2 will rise after a meal.

Together, the results discussed so far suggest that the plasma LEAP2 depends on both the long-term, underlying metabolic state (e.g., body mass and adiposity) as well as more short-term, meal-dependent changes in nutrient availability. The human studies suggest that plasma LEAP2 is highest in individuals with obesity after meals, possibly functioning as a nutritional "sufficiency hormone" that provides endocrine feedback to key brain regions, contributing to the feelings of satiety and satiation. Interestingly, the human studies demonstrated higher fasted plasma LEAP2 only in those with BMI greater than 35 to $40 \mathrm{~kg} / \mathrm{m}^{2}$, suggesting that milder degrees of obesity may not be associated with the "protective" elevations of LEAP2 and might benefit from therapies that would boost plasma LEAP2 (for instance, to curb appetite and reduce food reward). Similarly, therapies that increase plasma LEAP2 might also be beneficial particularly in individuals with obesity who have achieved weight loss, so as to counteract the naturally occurring falls in LEAP2 that otherwise may contribute to rebound weight gain, coincident with increases in plasma ghrelin $(69,70)$. Such LEAP2-based therapies could be impactful after lifestyle modification or gastric-banding surgery, in which there is no coincident beneficial increase in satiety gut hormones, such as peptide YY (PYY) and glucagon-like peptide 1, or decrease in acylghrelin, unlike after RYGB and/or VSG surgery.

The effects of oral glucose administration in mice were similar to those of food intake in mice and humans in increasing plasma LEAP2 and decreasing plasma acyl-ghrelin. These effects may therefore be driven by the presence of nutrients, and in particular glucose, in the gut or via associated increases in glucose or insulin in the systemic circulation or hepatic portal vein (52). Interestingly, insulin directly reduces stomach-derived ghrelin secretion (52). Our data suggest that blood glucose may itself regulate plasma LEAP2, independently of body mass or feeding status. Indeed, fasted plasma LEAP2 positively correlated with blood glucose and insulin resistance in a human cohort across a broad range of BMIs and with postprandial glucose, but not insulin, in another human cohort with obesity. Furthermore, the higher blood glucose in a T1DM mouse model of insulin deficiency was associated with higher plasma LEAP2, suggesting a more important role for increases in blood glucose than insulin to increase plasma LEAP2. It remains to be seen whether glucose administration-induced increases in plasma LEAP2 contribute to the blunted acyl-ghrelin orexigenic efficacy observed upon glucose administration (71).

Even though we found that plasma LEAP2 is regulated by body mass, acute and chronic nutritional status, and blood glucose in a manner opposite of plasma acyl-ghrelin in humans and mice in the settings discussed above, such reciprocal physiological regulation was not observed in obese individuals following VSG or in STZ-treated mice. In particular, as compared with presurgery levels, VSG reduced plasma LEAP2 levels (in the postprandial state, but not the fasted state). However, unlike the higher plasma acyl-ghrelin observed in normal weight humans (vs. humans with obesity) or following diet-induced weight-loss, fasted and postprandial acylghrelin concentrations after VSG-induced weight loss were lower than presurgery concentrations. Furthermore, in mice, STZ administration raised both plasma LEAP2 and acyl-ghrelin. This might be because of the loss of insulin-mediated suppression of ghrelin secretion after STZ (52). Thus, in both those conditions, the mean plasma LEAP2/acyl-ghrelin molar ratio remained unchanged and does not appear to fit within the model proposed in Figure 8. Further studies are required to address the potential impact of these VSG surgery- and STZ treatment-associated changes, as compared with the more usual (physiological) pattern of inverse regulation of LEAP2 and acyl-ghrelin, and in particular, whether this dysregulation could affect overall metabolic responses to endogenous or administered acyl-ghrelin in those settings.

Together, our results suggest a model (Figure 8) in which, during negative energy balance (acute fasting or longer-term energy restriction), a fall in plasma LEAP2 creates a permissive environment for elevated plasma acyl-ghrelin to most effectively act to increase food intake and $\mathrm{GH}$ secretion and prevent potentially life-threatening falls in blood glucose. Our model also predicts that in the settings of obesity, especially in the postprandial state in individuals with severe obesity, and/or raised blood glucose stemming from food intake, a coordinated rise in plasma LEAP2 and drop in plasma acyl-ghrelin limits acyl-ghrelin's orexigenic and blood glucose-raising actions. As plasma LEAP2 is positively correlated with BMI, we predict that individuals with milder forms of obesity, in particular, might benefit from potential weight-loss therapies that increase plasma LEAP2. So too would individuals who have achieved weight loss through lifestyle interventions, but run the risk of rebound weight gain, as weight loss induces falls in plasma LEAP2 and reciprocal increases in plasma acyl-ghrelin. These reciprocal increases and reductions in plasma LEAP2 and acyl-ghrelin observed upon weight loss from dieting and in several other key physiological states become uncoupled following VSG in humans or induction of T1DM in mice, although the mechanism of this uncoupling is not yet clear, nor is the functional significance. Furthermore, we predict that the plasma LEAP2/acyl-ghrelin ratio could be a key determinant modulating GHSR signaling in response to changes in body mass, acute and chronic nutritional states, and blood glucose.

\section{Methods}

Mouse studies. Male C57BL/6N mice (originally from Charles River Laboratories) bred and maintained in our colony were used. Mice were housed under a 12-hour dark/12-hour light cycle with free access to water and standard chow diet (2016 Teklad Global 16\% protein diet), unless otherwise indicated.

DIO and weight loss mouse models. Mice were weaned and individually housed at 3 weeks of age. At 4 weeks of age, mice were either maintained on standard chow diet or switched to HFD (Envigo Teklad TD88137; 42\% of kcals are fat derived) for 16 weeks. After 16 weeks, body composition analyses were performed using an EchoMRI-100 (Echo Medical Systems), and blood samples were collected for plasma LEAP2 and acyl-ghrelin measurement. The mice were sacrificed 2 weeks after the blood collection to harvest organs for quantitative RT-PCR. See Supplemental Methods for the RT-PCR protocol used. 
To model weight loss after obesity, a separate cohort of individually housed 4- to 5-week-old mice were fed HFD for 8 weeks to induce weight gain. Thereafter, the HFD-fed mice were either kept on HFD for 4 more weeks (obese group) or switched to standard chow for 4 weeks to induce weight loss (weight loss group). Mice fed standard chow during the entire study duration of 12 weeks served as controls (lean group). Body composition analysis and blood collection to measure plasma LEAP2 and acyl-ghrelin occurred at 8 weeks and again at 12 weeks. Body weight was measured weekly throughout the study duration.

T1DM mouse model. Eight- to ten-week-old mice were administered freshly prepared STZ $(150 \mathrm{mg} / \mathrm{kg}$ body weight, i.p.) in sodium citrate buffer as vehicle. Control mice were treated with vehicle. Blood was sampled 6 days later for measurement of blood glucose, plasma LEAP2, and acyl-ghrelin.

Human studies. Three human adult cohorts were used; details regarding participant characteristics are available in the Supplemental Methods. Cohort $1(n=90)$ was recruited as part of clinical research functional MRI (fMRI) studies at Imperial College London (Supplemental Figure 5). The participants included both sexes and individuals across diverse BMI categories. During a first visit, overnight-fasted venous blood samples were taken at approximately 10:30 am and 11:00 am (for plasma glucose, serum insulin, and triglyceride assays) and at 11:00 am (for LEAP2 and acyl-ghrelin assays); height and weight were measured to determine BMI; and percentage of body fat was determined by bioelectrical impedance analysis (Bodystat 1500). Nearly half the cohort $(n=41)$ had another visit within 1 to 2 weeks for whole-body MRI to determine VAT, SCAT, VAT/SCAT ratio, soleus and tibialis muscle IMCL, and IHCL, as described $(72,73)$. An additional $n=15$ participants were added to create cohort $1 \mathrm{Ex}(n=105$ adults), so as to include better representation of participants with obesity; during their single study visit, these additional participants had overnight-fasted venous blood samples taken for plasma LEAP2 measurement and BMI was determined.

Cohort $2(n=32)$ was recruited as part of a previously reported fMRI study (55) at UT Southwestern Medical Center and the Veterans Administration North Texas Health Care System (Supplemental Figure 8). The participants included age-matched women with either obesity $\left(\mathrm{BMI}>35 \mathrm{~kg} / \mathrm{m}^{2}\right)$ or normal weight $\left(\mathrm{BMI}<25 \mathrm{~kg} / \mathrm{m}^{2}\right)$. During their visit, overnight-fasted venous blood was collected, a standard meal of $337 \mathrm{kcal}$ (52\% carbohydrates, 30\% fat, 18\% protein) was consumed, and a postprandial blood sample was collected 1.5 hours after the start of meal ingestion. Though it was not published as part of the initial report (55), approximately 2 weeks after the initial study session visit, a subset of the cohort 2 women with obesity underwent a VSG procedure (cohort $2 \mathrm{~A}, n=7$ ). These participants returned for a second study session visit 12 to 18 months after VSG, during which the same protocol detailed above was performed.

Cohort $3(n=20)$ was recruited from bariatric clinics at the Imperial Weight Centre, St. Mary's Hospital, and the Chelsea and Westminster Hospital (London, United Kingdom) for clinical research studies at Imperial College London (Supplemental Figure 9). All individuals had obesity (BMI $>35 \mathrm{~kg} / \mathrm{m}^{2}$ ). During an initial postprandial visit, they arrived after eating a normal breakfast at home and baseline venous blood was sampled at approximately 12:00-1:00 pm (approximately 5 to 6 hours after breakfast). They consumed a $600 \mathrm{kcal}, 250 \mathrm{~mL}$ liquid meal (49\% carbohydrate, 35\% fat, $16 \%$ protein; Fortisip Compact Vanilla, Nutricia Advanced Medical Nutrition), and had repeat blood draws 1 hour and 2 hours after the standardized meal. During a second fasted visit approximately 1 week later, overnight-fasted venous blood samples were taken at approximately 1:00 pm. BMI and percentage of body fat were determined by bioelectrical impedance analysis (BC418, Tanita Europe VB). Two subsets of cohort 3 were also studied after RYGB surgery. Cohort 3A ( $n=14)$ was studied approximately 3 months after RYGB (and in $n=8$ participants, again at approximately 2 years after RYGB) by repeating the fasted visit described above. Cohort 3B ( $n=11)$ was studied approximately 3 months after RYGB (approximately 2 to 3 weeks after having returned to a solid diet) by repeating the postprandial visit described above.

Calculation of plasma LEAP2/acyl-ghrelin molar ratio. Plasma LEAP2 and acyl-ghrelin concentrations in $\mathrm{ng} / \mathrm{mL}$ were converted to molar concentrations by using the following formula: molar concentration $(\mathrm{mol} / \mathrm{L})=$ mass $(\mathrm{g}) /($ volume $[\mathrm{L}] \times$ molecular weight $[\mathrm{g} / \mathrm{mol}])$. The LEAP2/acyl-ghrelin molar ratio was calculated by dividing plasma LEAP2 molar concentration by plasma acyl-ghrelin molar concentration for each individual human or mouse. See Supplemental Methods for details on blood collection, blood processing, and assays used to measure LEAP2, acyl-ghrelin, blood glucose, and other analytes in the human and mouse samples.

Electrophysiology. See Supplemental Methods for details.

Statistics. Results are presented as dot plots or as box plots displaying median as a line within the box, interquartile range (IQR) as the box, $95 \% \mathrm{CI}$ as bars flanking the box, outliers as dots $(<5 \%,>95 \% \mathrm{CI})$, and mean as a plus sign. Within the main text, results are presented as mean \pm SEM, except as noted. The statistical tests ( 2 sided) used are indicated in the figure legends, and together with the graph preparations were performed using GraphPad Prism version 7.0.4 or version 8.1.0. Data sets not conforming to the assumption of normal distribution (Kolmogorov-Smirnov test, $P<0.05$ ) were analyzed by nonparametric tests. $P$ values of less than 0.05 were considered statistically significant, and $P$ values greater than or equal to 0.05 and less than 0.1 were considered evidence of statistical trends.

Study approval. The human studies were approved by the UT Southwestern Medical Center and the Veterans Administration North Texas Health Care System at Dallas Institutional Review Boards and United Kingdom Research Ethics Committees. The studies were carried out by principles outlined in the Declaration of Helsinki, and written informed consent was obtained from all participants. All animal procedures and use of mice were approved by the Institutional Animal Care and Use Committee of UT Southwestern Medical Center.

\section{Author contributions}

BKM, NP, KWW, APG, and JMZ conceptualized the experiments. BKM, NP, ZH, JAR, SOL, NPM, NC, BG, MOT, ELT, JDB, KWW, APG, and JMZ performed the experiments and/or analyzed data. $\mathrm{BKM}, \mathrm{ZH}, \mathrm{KWW}, \mathrm{APG}$, and JMZ wrote the manuscript. JMZ, JDB, APG, KWW, and NP secured funding. JMZ, APG, NP, and KWW supervised the research activity.

\section{Acknowledgments}

This work was supported by the NIH (R01DK103884 to JMZ, R01DK100699 and R01DK119169 to KWW, and NCATS ULTR000451 to NP), the Diana and Richard C. Strauss Professorship in Biomedical Research, the Mr. and Mrs. Bruce G. Brookshire Professorship in Medicine, the Kent and Jodi Foster Distinguished 
Chair in Endocrinology, in Honor of Daniel Foster, M.D., a gift from the David and Teresa Disiere Foundation (to JMZ), and funds from UT Southwestern Medical Center Department of Surgery (to NP). In the United Kingdom, this work was supported by grants from the United Kingdom Medical Research Council, the Wellcome Trust, the Imperial College Healthcare Charity, the European Union Sixth Framework Marie-Curie Programme, and an Imperial Wellcome-GSK fellowship (to NC), and infrastructure support was provided by the National Institute of Health Research (NIHR) Imperial Biomedical Research Centre and the NIHR Imperial Clinical Research Facility, Imperial College Healthcare National Health Service (NHS) Trust, London, United Kingdom. The views expressed are those of the authors and not necessarily those of the NHS, the NIHR, or the United Kingdom Department of Health and Social Care. We thank Joel Elmquist (UT Southwestern Medical Center) for kindly providing the NPY-hrGFP mice. We thank the staff of the Metabolic and Molecular Imaging Group and the Robert Steiner MRI Unit, MRC Clinical Sciences Centre,
Imperial College London; the Division of Diabetes, Endocrinology and Metabolism, Imperial College London; the NIHR Imperial Clinical Research Facility, Hammersmith Hospital; the Imperial Weight Centre, St. Mary's Hospital, the Imperial College Healthcare NHS Trust, and the Bariatric Clinic, Chelsea and Westminster Hospital NHS Foundation Trust, London, United Kingdom for assistance with the United Kingdom studies.

Address correspondence to: Jeffrey M. Zigman, University of Texas Southwestern Medical Center, 5323 Harry Hines Blvd., Dallas, Texas 75390-9077, USA. Phone: 214.648.6422; Email: jeffrey.zigman@ utsouthwestern.edu. Or to: Anthony P. Goldstone, Room E313, C3NL, 3rd Floor Burlington Danes Building, Imperial College London, Hammersmith Hospital, Du Cane Road. London W12 ONN, UK. Phone: 44.20.7594.5989; Email: tony.goldstone@imperial.ac.uk. Or to: Bharath K. Mani, University of Texas Southwestern Medical Center, 5323 Harry Hines Boulevard, Dallas, Texas 75390-9077, USA Phone:214.648.4709; Email: bharath.mani@utsouthwestern.edu.
1. Müller TD, et al. Ghrelin. Mol Metab. 2015;4(6):437-460.

2. Mani BK, Zigman JM. Ghrelin as a survival hormone. Trends Endocrinol Metab. 2017;28(12):843-854.

3. Kojima M, Hosoda H, Date Y, Nakazato M, Matsuo H, Kangawa K. Ghrelin is a growthhormone-releasing acylated peptide from stomach. Nature. 1999;402(6762):656-660.

4. Zigman JM, Jones JE, Lee CE, Saper CB, Elmquist JK. Expression of ghrelin receptor mRNA in the rat and the mouse brain. JComp Neurol. 2006;494(3):528-548

5. Yang J, Brown MS, Liang G, Grishin NV, Goldstein JL. Identification of the acyltransferase that octanoylates ghrelin, an appetite-stimulating peptide hormone. Cell. 2008;132(3):387-396.

6. Gutierrez JA, et al. Ghrelin octanoylation mediated by an orphan lipid transferase. Proc Natl Acad Sci US A. 2008;105(17):6320-6325.

7. Toshinai K, et al. Des-acyl ghrelin induces food intake by a mechanism independent of the growth hormone secretagogue receptor. Endocrinology. 2006;147(5):2306-2314.

8. Broglio F, et al. Non-acylated ghrelin counteracts the metabolic but not the neuroendocrine response to acylated ghrelin in humans. JClin Endocrinol Metab. 2004;89(6):3062-3065.

9. Delhanty PJ, Neggers SJ, van der Lely AJ. Des-acyl ghrelin: a metabolically active peptide. Endocr Dev. 2013;25:112-121.

10. Ariyasu $\mathrm{H}$, et al. Stomach is a major source of circulating ghrelin, and feeding state determines plasma ghrelin-like immunoreactivity levels in humans. J Clin Endocrinol Metab. 2001;86(10):4753-4758.

11. Tschöp M, Smiley DL, Heiman ML. Ghrelin induces adiposity in rodents. Nature. 2000;407(6806):908-913.

12. Asakawa A, et al. Ghrelin is an appetite-stimulatory signal from stomach with structural resemblance to motilin. Gastroenterology. 2001;120(2):337-345.

13. Mani BK, Osborne-Lawrence S, Vijayaraghavan
P, Hepler C, Zigman JM. $\beta 1$-Adrenergic receptor deficiency in ghrelin-expressing cells causes hypoglycemia in susceptible individuals. J Clin Invest. 2016;126(9):3467-3478.

14. Mani BK, et al. Hypoglycemic effect of combined ghrelin and glucagon receptor blockade. Diabetes. 2017;66(7):1847-1857.

15. Liu J, et al. Novel ghrelin assays provide evidence for independent regulation of ghrelin acylation and secretion in healthy young men. JClin Endocrinol Metab. 2008;93(5):1980-1987.

16. Li RL, Sherbet DP, Elsbernd BL, Goldstein JL, Brown MS, Zhao TJ. Profound hypoglycemia in starved, ghrelin-deficient mice is caused by decreased gluconeogenesis and reversed by lactate or fatty acids. J Biol Chem. 2012;287(22):17942-17950.

17. Zhang Y, Fang F, Goldstein JL, Brown MS, Zhao TJ. Reduced autophagy in livers of fasted, fat-depleted, ghrelin-deficient mice: reversal by growth hormone. Proc Natl Acad Sci U S A. 2015;112(4):1226-1231.

18. Cummings DE, Purnell JQ, Frayo RS, Schmidova $\mathrm{K}$, Wisse BE, Weigle DS. A preprandial rise in plasma ghrelin levels suggests a role in meal initiation in humans. Diabetes. 2001;50(8):1714-1719.

19. Briggs DI, Andrews ZB. Metabolic status regulates ghrelin function on energy homeostasis. Neuroendocrinology. 2011;93(1):48-57.

20. Wren AM, et al. Ghrelin causes hyperphagia and obesity in rats. Diabetes. 2001;50(11):2540-2547.

21. Kamegai J, Tamura H, Shimizu T, Ishii S, Sugihara $\mathrm{H}$, Wakabayashi I. Chronic central infusion of ghrelin increases hypothalamic neuropeptide $\mathrm{Y}$ and Agouti-related protein mRNA levels and body weight in rats. Diabetes. 2001;50(11):2438-2443.

22. Sun Y, Wang P, Zheng H, Smith RG. Ghrelin stimulation of growth hormone release and appetite is mediated through the growth hormone secretagogue receptor. Proc Natl Acad Sci U S A. 2004;101(13):4679-4684.

23. Zigman JM, et al. Mice lacking ghrelin receptors resist the development of diet-induced obesity. JClin Invest. 2005;115(12):3564-3572.
24. Wortley KE, et al. Genetic deletion of ghrelin does not decrease food intake but influences metabolic fuel preference. Proc Natl Acad Sci US A. 2004;101(21):8227-8232.

25. Zhao TJ, et al. Ghrelin O-acyltransferase (GOAT) is essential for growth hormone-mediated survival of calorie-restricted mice. Proc Natl Acad Sci US A. 2010;107(16):7467-7472.

26. Sun Y, Ahmed S, Smith RG. Deletion of ghrelin impairs neither growth nor appetite. Mol Cell Biol. 2003;23(22):7973-7981.

27. De Smet B, et al. Energy homeostasis and gastric emptying in ghrelin knockout mice. J Pharmacol Exp Ther. 2006;316(1):431-439.

28. Kirchner H, et al. GOAT links dietary lipids with the endocrine control of energy balance. Nat Med. 2009;15(7):741-745.

29. Pfluger PT, et al. Simultaneous deletion of ghrelin and its receptor increases motor activity and energy expenditure. Am J Physiol Gastrointest Liver Physiol. 2008;294(3):G610-G618.

30. Perreault M, Istrate N, Wang L, Nichols AJ, Tozzo E, Stricker-Krongrad A. Resistance to the orexigenic effect of ghrelin in dietary-induced obesity in mice: reversal upon weight loss. Int JObes Relat Metab Disord. 2004;28(7):879-885.

31. Naznin F, et al. Diet-induced obesity causes peripheral and central ghrelin resistance by promoting inflammation. J Endocrinol. 2015;226(1):81-92.

32. Gardiner JV, et al. The hyperphagic effect of ghrelin is inhibited in mice by a diet high in fat. Gastroenterology. 2010;138(7):2468-2476.e1.

33. Briggs DI, Enriori PJ, Lemus MB, Cowley MA, Andrews ZB. Diet-induced obesity causes ghrelin resistance in arcuate NPY/AgRP neurons. Endocrinology. 2010;151(10):4745-4755.

34. Zigman JM, Bouret SG, Andrews ZB. Obesity impairs the action of the neuroendocrine ghrelin system. Trends Endocrinol Metab. 2016;27(1):54-63.

35. McFarlane MR, Brown MS, Goldstein JL, Zhao TJ. Induced ablation of ghrelin cells in adult mice does not decrease food intake, body 
weight, or response to high-fat diet. Cell Metab. 2014;20(1):54-60.

36. Wang $\mathrm{Q}$, et al. Arcuate AgRP neurons mediate orexigenic and glucoregulatory actions of ghrelin. Mol Metab. 2014;3(1):64-72.

37. Brial F, Lussier CR, Belleville K, Sarret P, Boudreau F. Ghrelin inhibition restores glucose homeostasis in hepatocyte nuclear factor-1 $\alpha$ (MODY3)-deficient mice. Diabetes. 2015;64(9):3314-3320.

38. Ishii S, Kamegai J, Tamura H, Shimizu T, Sugihara $\mathrm{H}$, Oikawa S. Role of ghrelin in streptozotocininduced diabetic hyperphagia. Endocrinology. 2002;143(12):4934-4937.

39. Dong J, et al. Role of endogenous ghrelin in the hyperphagia of mice with streptozotocin-induced diabetes. Endocrinology. 2006;147(6):2634-2642.

40. Verhulst PJ, et al. Role of ghrelin in the relationship between hyperphagia and accelerated gastric emptying in diabetic mice. Gastroenterology. 2008;135(4):1267-1276.

41. Ge X, et al. LEAP2 is an endogenous antagonist of the ghrelin receptor. Cell Metab. 2018;27(2):461-469.e6.

42. Krause A, et al. Isolation and biochemical characterization of LEAP-2, a novel blood peptide expressed in the liver. Protein Sci. 2003;12(1):143-152.

43. Howard A, Townes C, Milona P, Nile CJ, Michailidis G, Hall J. Expression and functional analyses of liver expressed antimicrobial peptide-2 (LEAP-2) variant forms in human tissues. Cell Immunol. 2010;261(2):128-133.

44. Henriques ST, Tan CC, Craik DJ, Clark RJ. Structural and functional analysis of human liverexpressed antimicrobial peptide 2. Chembiochem. 2010;11(15):2148-2157.

45. Cowley MA, et al. The distribution and mechanism of action of ghrelin in the CNS demonstrates a novel hypothalamic circuit regulating energy homeostasis. Neuron. 2003;37(4):649-661.

46. Chen SR, et al. Ghrelin receptors mediate ghrelin-induced excitation of agouti-related protein/neuropeptide $\mathrm{Y}$ but not pro-opiomelanocortin neurons. J Neurochem. 2017;142(4):512-520.

47. Nakazato M, et al. A role for ghrelin in the central regulation of feeding. Nature. 2001;409(6817):194-198.

48. Shintani M, et al. Ghrelin, an endogenous growth hormone secretagogue, is a novel orexigenic pep- tide that antagonizes leptin action through the activation of hypothalamic neuropeptide Y/Y1 receptor pathway. Diabetes. 2001;50(2):227-232.

49. Toshinai K, et al. Upregulation of Ghrelin expression in the stomach upon fasting, insulin-induced hypoglycemia, and leptin administration. Biochem Biophys Res Commun. 2001;281(5):1220-1225.

50. Greenman Y, Golani N, Gilad S, Yaron M, Limor $\mathrm{R}$, Stern N. Ghrelin secretion is modulated in a nutrient- and gender-specific manner. Clin Endocrinol (Oxf). 2004;60(3):382-388.

51. Foster-Schubert KE, et al. Acyl and total ghrelin are suppressed strongly by ingested proteins, weakly by lipids, and biphasically by carbohydrates. JClin Endocrinol Metab. 2008;93(5):1971-1979.

52. Mani BK, Shankar K, Zigman JM. Ghrelin's relationship to blood glucose. Endocrinology. 2019;160(5):1247-1261.

53. Gelling RW, et al. Effect of uncontrolled diabetes on plasma ghrelin concentrations and ghrelin-induced feeding. Endocrinology. 2004;145(10):4575-4582.

54. Masaoka T, et al. Enhanced plasma ghrelin levels in rats with streptozotocin-induced diabetes. FEBS Lett. 2003;541(1-3):64-68.

55. Puzziferri N, et al. Brain imaging demonstrates a reduced neural impact of eating in obesity. Obesity (Silver Spring). 2016;24(4):829-836.

56. Cummings DE, et al. Plasma ghrelin levels after diet-induced weight loss or gastric bypass surgery. N EnglJMed. 2002;346(21):1623-1630.

57. Shiiya $\mathrm{T}$, et al. Plasma ghrelin levels in lean and obese humans and the effect of glucose on ghrelin secretion. JClin Endocrinol Metab. 2002;87(1):240-244.

58. Tschöp M, Weyer C, Tataranni PA, Devanarayan V, Ravussin E, Heiman ML. Circulating ghrelin levels are decreased in human obesity. Diabetes. 2001;50(4):707-709.

59. Mondal MS, et al. Identification of ghrelin and its receptor in neurons of the rat arcuate nucleus. Regul Pept. 2005;126(1-2):55-59.

60. Willesen MG, Kristensen P, Rømer J. Co-localization of growth hormone secretagogue receptor and NPY mRNA in the arcuate nucleus of the rat. Neuroendocrinology. 1999;70(5):306-316.

61. Chen HY, et al. Orexigenic action of peripheral ghrelin is mediated by neuropeptide $\mathrm{Y}$ and agouti-related protein. Endocrinology. 2004;145(6):2607-2612.
62. M'Kadmi C, et al. N-terminal liver-expressed antimicrobial peptide 2 (LEAP2) region exhibits inverse agonist activity toward the ghrelin receptor. J Med Chem. 2019;62(2):965-973.

63. Wang JH, et al. Identifying the binding mechanism of LEAP2 to receptor GHSR1a. FEBS J. 2019;286(7):1332-1345.

64. Sun Y, Butte NF, Garcia JM, Smith RG. Characterization of adult ghrelin and ghrelin receptor knockout mice under positive and negative energy balance. Endocrinology. 2008;149(2):843-850.

65. Kamegai J, Tamura H, Shimizu T, Ishii S, Sugihara $\mathrm{H}$, Wakabayashi I. Central effect of ghrelin, an endogenous growth hormone secretagogue, on hypothalamic peptide gene expression. Endocrinology. 2000;141(12):4797-4800.

66. Andrews ZB. Central mechanisms involved in the orexigenic actions of ghrelin. Peptides. 2011;32(11):2248-2255.

67. Martin NM, Small CJ, Sajedi A, Patterson M, Ghatei MA, Bloom SR. Pre-obese and obese agouti mice are sensitive to the anorectic effects of peptide YY(3-36) but resistant to ghrelin. Int $J$ Obes Relat Metab Disord. 2004;28(7):886-893.

68. Briggs DI, et al. Evidence that diet-induced hyperleptinemia, but not hypothalamic gliosis, causes ghrelin resistance in NPY/AgRP neurons of male mice. Endocrinology. 2014;155(7):2411-2422.

69. Briggs DI, Lockie SH, Wu Q, Lemus MB, Stark R, Andrews ZB. Calorie-restricted weight loss reverses high-fat diet-induced ghrelin resistance, which contributes to rebound weight gain in a ghrelin-dependent manner. Endocrinology. 2013;154(2):709-717.

70. Chen VP, Gao Y, Geng L, Brimijoin S. Butyrylcholinesterase gene transfer in obese mice prevents postdieting body weight rebound by suppressing ghrelin signaling. Proc Natl Acad Sci U S A. 2017;114(41):10960-10965.

71. Lockie SH, et al. Glucose availability predicts the feeding response to ghrelin in male mice, an effect dependent on AMPK in AgRP neurons. Endocrinology. 2018;159(11):3605-3614.

72. Goldstone AP, et al. Elevated fasting plasma ghrelin in prader-willi syndrome adults is not solely explained by their reduced visceral adiposity and insulin resistance. JClin Endocrinol Metab. 2004;89(4):1718-1726.

73. Thomas EL, et al. The missing risk: MRI and MRS phenotyping of abdominal adiposity and ectopic fat. Obesity (Silver Spring). 2012;20(1):76-87. 